Portland State University

PDXScholar

$1-29-1993$

\title{
Dynamics of Ultrashort Pulse Generation and Amplification in Dye Lasers
}

Shuanghua Jiang

Portland State University

Follow this and additional works at: https://pdxscholar.library.pdx.edu/open_access_etds

Part of the Electrical and Computer Engineering Commons Let us know how access to this document benefits you.

Recommended Citation

Jiang, Shuanghua, "Dynamics of Ultrashort Pulse Generation and Amplification in Dye Lasers" (1993). Dissertations and Theses. Paper 1310.

https://doi.org/10.15760/etd.1309

This Dissertation is brought to you for free and open access. It has been accepted for inclusion in Dissertations and Theses by an authorized administrator of PDXScholar. Please contact us if we can make this document more accessible: pdxscholar@pdx.edu. 


\title{
DYNAMICS OF ULTRASHORT PULSE GENERATION AND AMPLIFICATION IN DYE LASERS
}

\author{
by \\ SHUANGHUA JIANG
}

\begin{abstract}
A dissertation submitted in partial fulfillment of the requirements for the degree of

\section{DOCTOR OF PHILOSOPHY}

in

ELECTRICAL AND COMPUTER ENGINEERING
\end{abstract}

Portland State University

1993 


\section{TO THE OFFICE OF GRADUATE STUDIES:}

The members of the Committee approve the dissertation of Shuanghua Jiang presented January 29, 1993.

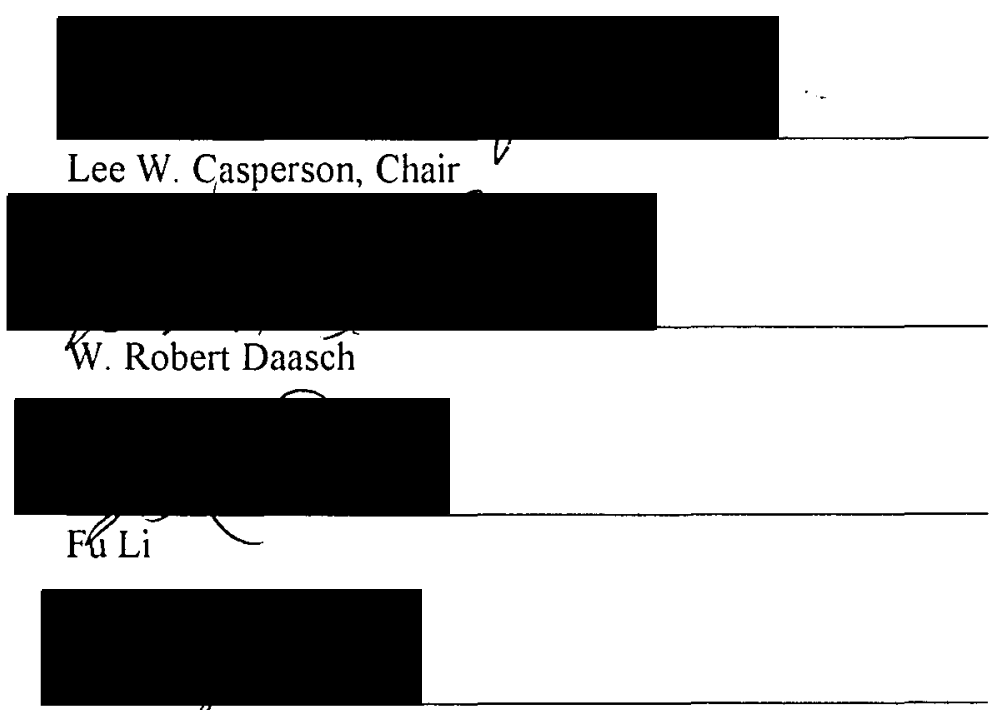

Richard'Tymerski

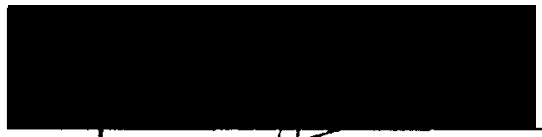

Erik Bodegont

APPROVED:

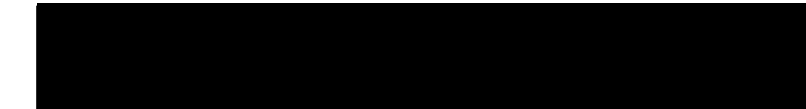

Rolf Schaumann, Chair, Department of Electrical Engineering

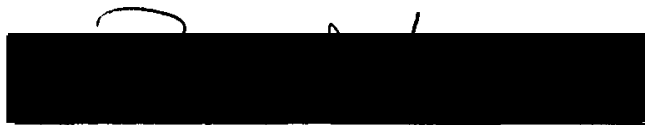

Roy W. Koch, Vice Provost for Graduate Studies and Research 
AN ABSTRACT OF THE DISSERTATION OF Shuanghua Jiang for the Doctor of Philosophy in Electrical and Computer Engineering presented January 29, 1993.

Title: Dynamics of Ultrashort Pulse Generation and Amplification in Dye Lasers.

APPROVED BY THE MEMBERS OF THE DISSERTATION COMMITTEE:
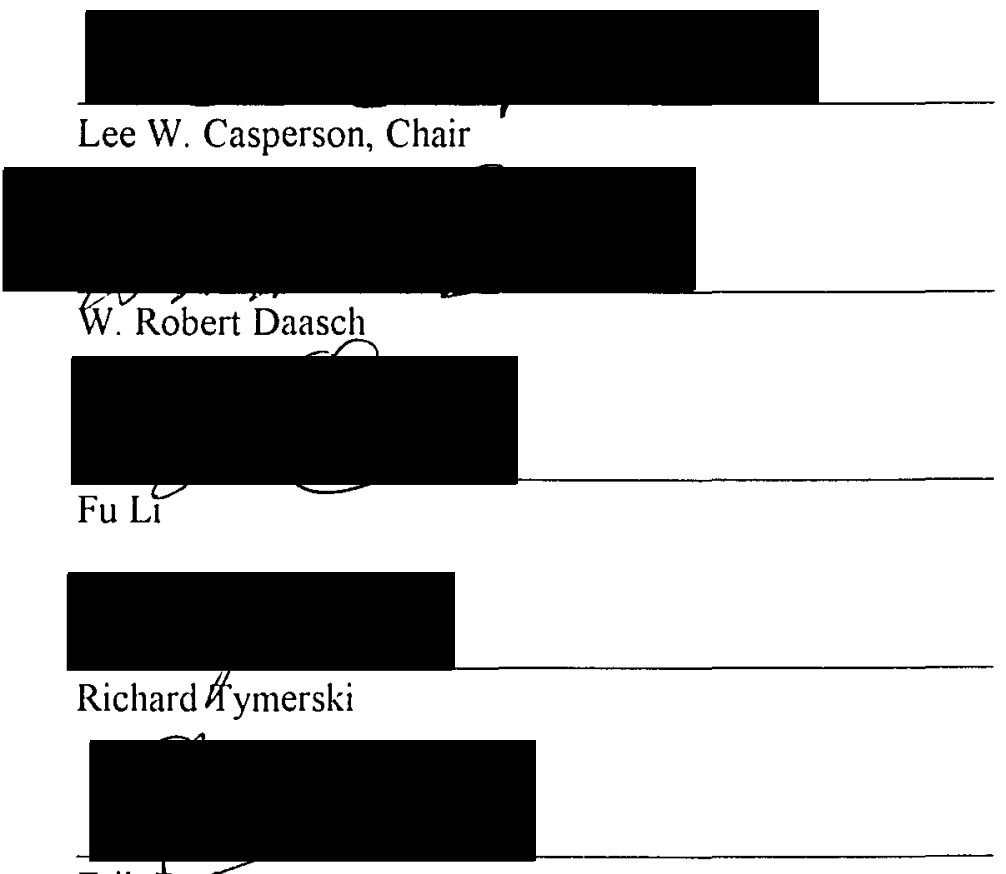

Erik Bođegom

The dynamics of ultrashort pulse generation and amplification in dye lasers is studied in this dissertation. In particular, we have developed general semiclassical models for ultrashort pulse dye laser amplifiers and oscillators. These models start from Maxwell's equation for the electric field and density matrix equations for the active laser medium. A finite coherence time or phase memory time of the molecular wave functions, a finite vibrational relaxation time for the lower electronic state of the dye laser transition, 
an isotropic molecular orientational distribution, and an arbitrary pump polarization are all taken into account. Based on these models, specific topics that are discussed herein include pump polarization effects, timing and detuning studies in synchronously pumped mode-locked dye lasers, and amplification of ultrashort pulses in dye laser amplifiers. Properties such as pulse width, pulse shape, pulse intensity, pulse stability, pulse amplification efficiency, etc., are studied in detail. 


\section{ACKNOWLEDGMENT}

I have had the good fortune to have interacted with a large number of people who have offered friendship and assistance throughout my study here at Portland State. I must, in particular, thank Professor Lee W. Casperson, who taught me not only about lasers but

also ways to handle nonacademic matters. A special thanks, too, goes to my parents in China for their constant care and encouragement. I am satisfied on those occasions when I make them proud. Above all, I am grateful to my wife Ali for her unconditional support, patience, and encouragement.

I also wish to acknowledge financial support during the course of this work from the National Science Foundation and the Jorgenson Scholarship for Science. 
TABLE OF CONTENTS

PAGE

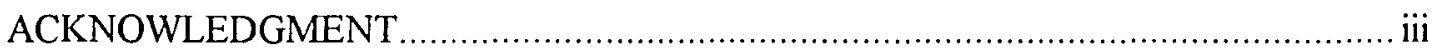

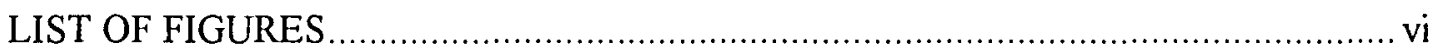

\section{CHAPTER}

I INTRODUCTION....................................................................

II THEORY OF ULTRASHORT PULSE DYE LASERS .......................4

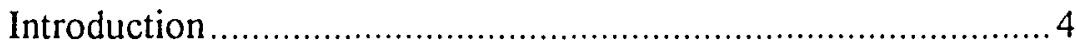

Theory of Ultrashort Pulse Dye Laser Amplifiers....................... 6

Longitudinal Pumping

Transverse Pumping

Rate Equation Approximation

Unidirectional Molecular Distribution Model

Theory of Synchronously Pumped Mode-Locked Dye

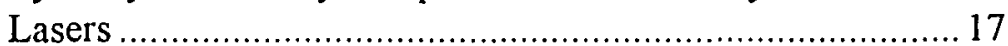

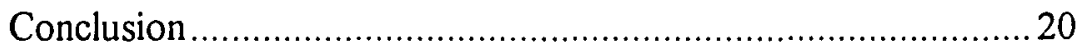

III PUMP POLARIZATION EFFECTS IN SYNCHRONOUSLY

PUMPED MODE-LOCKED DYE LASERS ....................................2 22

Introduction ................................................................ 22

Numerical Results ....................................................... 24

Experimental Results.................................................. 31

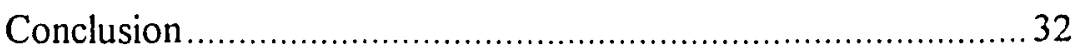

IV TIMING AND DETUNING STUDIES OF A

SYNCHRONOUSLY MODE-LOCKED DYE LASER ......................35 


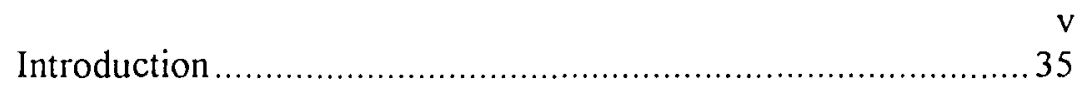

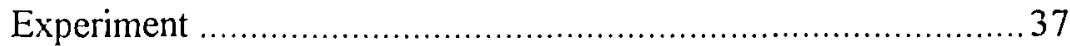

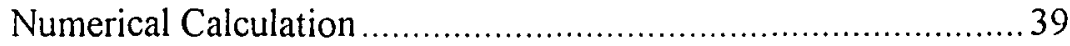

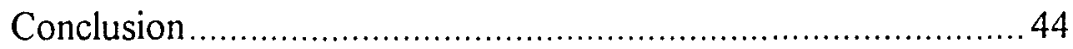

V ULTRASHORT PULSE PROPAGATION IN DYE LASER

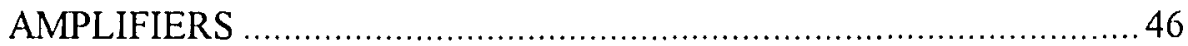

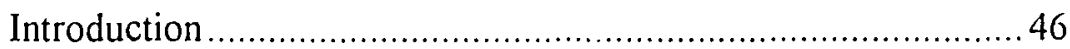

Simplifications of the Theory ................................................ 48

Semiclassical Model

Simplifications

Numerical Results ............................................................. 51

General Results

Rate Equation Approximation

Unidirectional Molecular Distribution

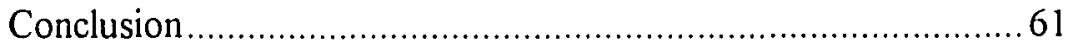

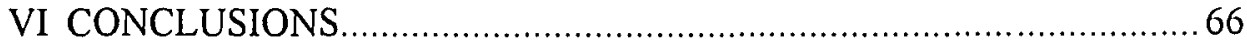

REFERENCES 


\section{LIST OF FIGURES}

\section{FIGURE}

PAGE

1. Energy level model used in the dye laser analysis.

2. Geometry of pump and signal fields and molecular dipole moments in the longitudinal pump arrangement

3. Geometry of pump and signal fields and molecular dipole moments in the transverse pump arrangement

4. Temporal traces of the output pulses with pump polarization angles of $0^{\circ}, 20^{\circ}$, and $30^{\circ}$ for the threshold parameters (a) $r=1.3$, (b) 1.46 , and (c) 4.0

5. Intensity autocorrelations of the output pulses with pump polarization angles of $0^{\circ}, 20^{\circ}$, and $30^{\circ}$ for the threshold parameters (a) $r=$ 1.3 , (b) 1.46 , and (c) 4.0 .

6. Theoretical pulse-width vs. the pump polarization angle for the threshold parameters $r=1.3,1.46,4.0$, and 10.0 .

7. Output pulse peak intensities vs. pump polarization angle for the threshold parameters $r=1.3,1.46$, and 4.0 .

8. Normalized average power of the output pulses in polar form.

9. Peak-to-peak time delay between the pump pulse and the signal pulse versus the pump polarization angle for threshold parameter values of $r=1.3,1.46$, and 4.0 .

10. Schematic of the experimental set-up for study of the timing characteristics of the SPML dye laser (AC, autocorrelator; $D$, detector; RF, RF driver)

11. Sampling scope temporal traces (100 ps/div) of the mode-locked dye lasers for $r=1.7$ at various cavity length detunings.

12. Experimental, numerical dependence of the SPML dye laser pulse occurrence time on the cavity length detuning 
13. Schematic diagram of a pulse propagating in a dye laser amplifier and the gain process by depletion of stored energy; also illustrated are the evolution in time $\tau$ and space $\zeta$

14. Normalized intensity temporal profiles of amplified pulses with initial small signal gain-length product from 10 to 50 based on the general semiclassical model (solid lines) given in Eqs. (87)-(90) and rate equation model (dotted lines) given in Eqs. (106)-(108)......... 56

15. Normalized integrated (over the entire solid angle) population differences as a function of time.

16. Integrated pulse energy gain based on the general semiclassical model (a) and rate equation model (b) as a function of small signal gainlength product for input pulse widths of $1 \times 10^{-12} \mathrm{sec}$ (solid lines), $1 \times 10^{-13} \mathrm{sec}$ (dashed lines), and $1 \times 10^{-14} \mathrm{sec}$ (dotted lines)

17. Normalized intensity temporal profiles of amplified pulses with initial small signal gain-length products from 10 to 50 based on the general (isotropic molecular distribution) semiclassical model (solid lines) given in Eqs. (87)-(90) and the unidirectional molecular distribution model (dotted lines) given in Eqs. (109)(112).

18. Normalized integrated (over the entire solid angle) population differences as a function of time

19. Integrated pulse energy gain based on the unidirectional molecular distribution model of Eqs. (109)-(112) as a function of small signal gain-length product for input pulse widths of $1 \times 10^{-12} \mathrm{sec}$ (solid lines), $1 \times 10^{-13} \mathrm{sec}$ (dashed lines), and $1 \times 10^{-14} \mathrm{sec}$ (dotted lines). 


\section{CHAPTER I}

\section{INTRODUCTION}

Dye lasers are perhaps the most versatile and one of the most successful laser sources known today. Indeed, at the time of the discovery of this class of lasers by Sorokin and Lankard [1], few could have anticipated their spectacular diversification and their significant contribution to basic physics, chemistry, biology, and additional fields.

Dye lasers can operate in both pulsed and continuous wave (cw) conditions that are tunable from the near-UV to near-IR. Dynamics has been a issue to study in laser systems, and of course, is not limited to dye lasers. In general, dynamics means motion. Dynamics addresses the forces at work in a system, specially, how they actively interplay and change. At the root of all dynamics is a flow or exchange of energy. For example, in mechanical systems, we encounter forces that change the momentum and energy of particles in the system [2].

A laser is a special optical transducer. A laser converts energy supplied by a pump source into coherent light. When we design lasers, we seek both this energy conversion and to control the coherence properties of the light. By studying and modeling the dynamics of a laser, we can determine the absolute limits that we can achieve. In this dissertation, we will study the dynamics of one class of the dye laser--ultrashort pulsed dye lasers.

Depending on the particular attributes of the system at hand, these lasers produce wavelength tunable pulses in the range of $10^{-14} \mathrm{sec}$ to $10^{-12} \mathrm{sec}$. These ultrashort optical pulses have found extensive applications in areas such as spectroscopy and 
photochemistry, electronic material and electronic device characterization, electro-optic and opto-optic sampling, optical communication and optical computing. Driven by the needs of ultrashort pulse applications, some researchers seek the ways to produce stable pulses with shorter and shorter pulsewidth at high repetition rate. On the other hand, means to amplify pulses to higher and higher pulse energy are also sought. In general, stable and short pulses with high energy are most desirable.

This dissertation intends to investigate the dynamics of such ultrashort pulsed dye lasers concerning the pulse production and amplification. Even though the gain bandwidths of dye media used in the ultrashort dye lasers are wider compared to other laser active media, they are not infinite. As the limit of the bandwidth is approached, dynamic properties such as pulse widths, peak intensity, stability, amplification efficiency, etc. ought to be understood.

In Chapter II, a comprehensive semiclassical model governing the dynamics of ultrashort pulse dye laser amplifiers and oscillators is derived in detail. This model started from density matrix equations for the dye laser medium and Maxwell equation for the electric field. The model includes isotropic molecular distribution, a finite vibrational relaxation time, a finite coherence time, and an arbitrary pump polarization misalignment. It is believed that this is the most general model of ultrashort pulse dye lasers to date. In addition, corresponding simplified models such as a rate equation model and a unidirectional distribution model are also derived. Based on models developed in Chapter II, we have, in Chapter III, investigated the pump polarization effects in synchronously pumped mode-locked dye lasers, which is one class of most reliable ultrashort pulse dye lasers. Effects on pulse width, pulse shape, pulse intensity, etc. are included. In Chapter IV, we have addressed the problem of timing and detuning of synchronously pumped mode-locked dye lasers. Pulse occurrence time as a function of the cavity length 
mismatch is studied in detail both theoretically and experimentally. The issue of ultrashort pulse amplification by dye laser amplifiers is investigated in Chapter V. The validity of conventional models is discussed when applied to ultrashort pulse amplification. Coherence effects are found to play crucial roles in modeling the amplification of ultrashort pulses in dye amplifiers. Results obtained from the semiclassical model lead to important guidelines for the design of the ultrashort pulse dye laser amplifiers. 


\section{CHAPTER II}

\section{THEORY OF ULTRASHORT PULSE DYE LASERS}

\section{INTRODUCTION}

It was not until the synchronously pumped mode-locked dye laser produced intense, stable picosecond optical pulses that extensive theoretical investigations were begun [3]. The synchronous pumping concept was first demonstrated in 1968 using a pulsed pump source [4-6]. However, the technique remained of limited practical value until cw mode-locked pump sources were exploited [7, 8]. The first theoretical studies [9, 10] of synchronous pumping of mode-locked dye lasers were based on a model of a twolevel active medium with zero coherence time, or dephasing time of the dye laser media. Based on this model, further analyses were used to explain the synchronously pumped mode-locking process [11-15]. In some cases, these analyses have provided explicit results for the pulse shapes as functions of the various laser parameters. Initially the results obtained by this model fully satisfied the investigators, but gradually it has become clear that this approach does not describe accurately the development of synchronous lasing in picosecond or subpicosecond lasers.

Due to the inaccuracy and difficulties of the rate equation model mentioned above, multiple levels of the active medium with a transient polarization of the laser transition, or the semiclassical model have been developed [16-18]. This has led to a much better understanding of the physics of synchronous pumping and the formation of ultrashort pulses, and has made it possible to determine the ultimate potential of ultrashort dye lasers. As the semiclassical model employs a Maxwell-Schrödinger equation set 
governing the interaction of the pump and signal fields with the dye laser medium, it has several advantages over the more conventional rate equation discussions [16].

First, it may be noted that in the semiclassical model, the electric fields are vectors and one can account in a rigorous way for the fact that the emission and absorption dipoles of the isotropically distributed dye molecules are not generally parallel to the pump and signal fields.

Second, the semiclassical model includes the nonzero phase memory time, or coherence time of the molecular wave functions. Since this coherence time is known to be in the range of $10^{-14}$ to $10^{-12} \mathrm{sec}$, at least for rhodamine-based lasers, it would reasonable to expect that the finite phase memory time could have at most a minor effects on the evolution of picosecond mode-locked pulsation. However, the studies on synchronously pumped mode-locked dye lasers $[16,19,20]$ have shown that inclusion of coherence effects is essential to understanding the dye laser dynamics when the cavity length mismatch is very small. Coherence effects were also found to be significant for understanding ultrashort pulse evolution in excimer lasers [21-24]. As the optical pulses produced or amplified by dye lasers become shorter and shorter, coherence effects are expected to be more substantial. For example, 200 fsec pulses were obtained directly from a dye laser synchronously pumped by a compressed second harmonic of Nd:YAG laser [25], and $26 \mathrm{fsec}$ optical pulses were amplified by dye medium [26].

Another feature of the multi-level semiclassical model is that it includes the finite vibrational relaxation time in the lower electronic state of the dye laser transition. The vibrational relaxation time is on the order of one picosecond, and the inclusion of this effect was shown to be of great importance in interpreting the evolution of the picosecond laser pulses in the synchronously pumped mode-locked dye lasers [16]. It seems always 
necessary to include this finite relaxation time when one deals with picosecond, subpicosecond, or femtosecond pulsation in dye lasers.

Starting from Maxwell's equations for the signal and pump electric fields, and density matrix equations for a four-level dye laser medium, reference [16] developed a general model governing the light-matter interaction in dye lasers. This model assumed parallel pump and signal field polarization in a longitudinal pump arrangement. In the following sections, we will, based on the theory developed in reference [16], derive a semiclassical model for both longitudinal and transverse pumping configurations with arbitrary pump and signal field polarization. A corresponding rate equation model and a more conventional unidirectional molecular distribution model are also derived. Finally, this model is adopted to the synchronous pumping configuration by some proper simplifications.

\section{THEORY OF ULTRASHORT PULSE DYE LASER AMPLIFIERS}

The energy level model used in this treatment is the four-level system shown schematically in Figure 1. The pump absorption occurs between level 0 and level 3, while the signal stimulated emission takes place between levels 2 and 1. The molecules in level 3 decay nonradiatively to level 2 with a vibrational relaxation time $\tau_{3}$, while molecules in level 1 have a vibrational relaxation time $\tau_{1}$. The spontaneous decay time for the laser transition is represented by $\tau_{2}$. Setting up the density matrix equations and combining with Maxwell's wave equation, and making some reasonable simplifications and specializations, leads to a basic set of equations governing the interaction of short light pulses and matter in a dye laser medium. These equations were derived as [16]:

$\frac{\partial \rho_{22}}{\partial t}=\frac{1}{\hbar} \eta_{s}^{\prime} \mathbf{E}_{s}^{\prime} \cdot \mathbf{e}_{s}-\frac{\rho_{22}}{\tau_{2}}+\frac{T_{p}\left|\mu_{p}\right|^{2}}{2 \hbar^{2}}\left(\mathbf{E}_{\mathbf{s}}^{\prime} \cdot \mathbf{e}_{\mathrm{p}}\right)^{2}$ 


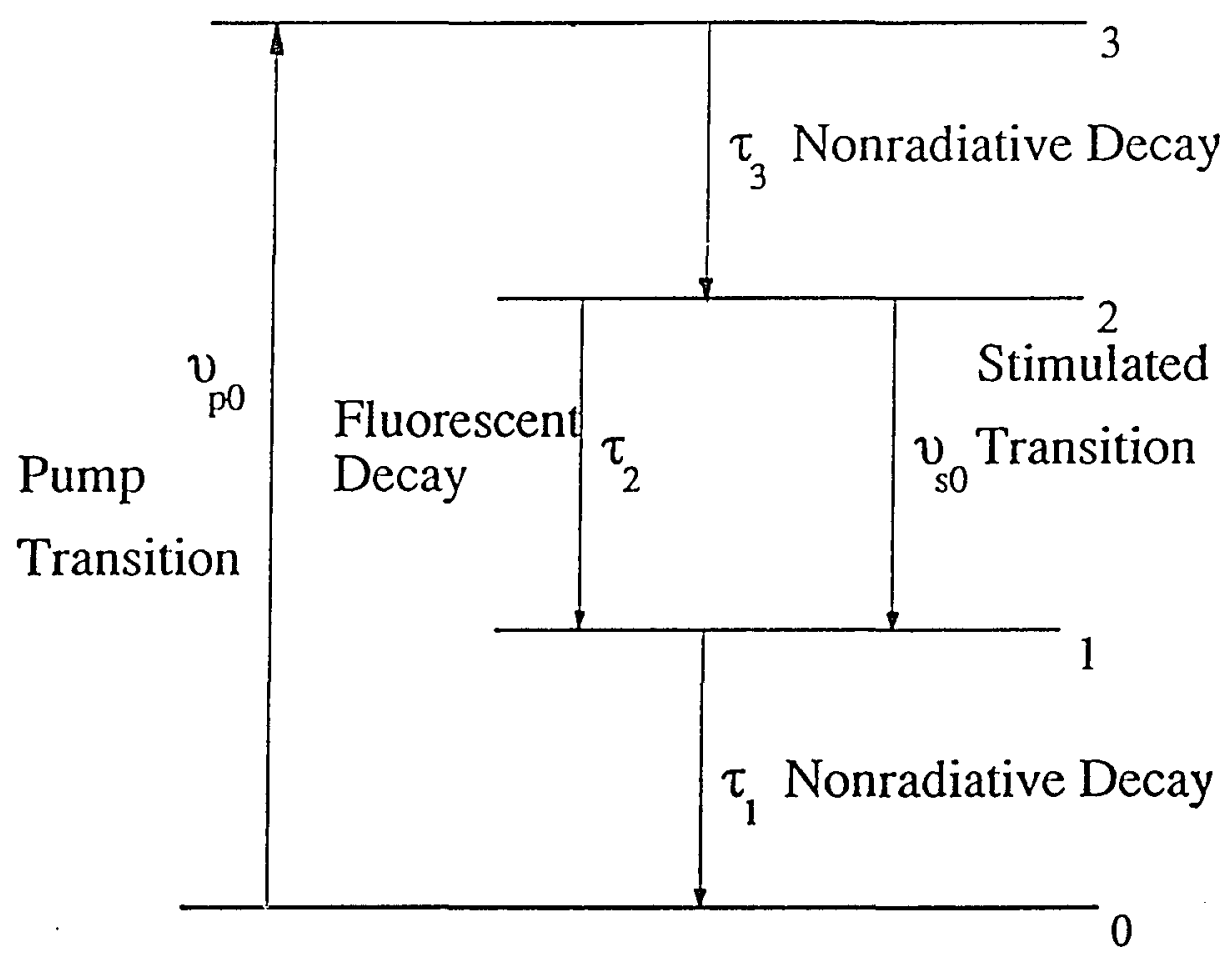

Figure 1. Energy level model used in the dye laser analysis. 
$\frac{\partial \rho_{11}}{\partial t}=-\frac{1}{\hbar} \eta_{s}^{\prime} \mathbf{E}_{\mathrm{s}}^{\prime} \cdot \mathbf{e}_{\mathrm{s}}-\frac{\rho_{11}}{\tau_{1}}+\frac{\rho_{22}}{\tau_{2}}$

$\frac{\partial \eta_{s}^{\prime}}{\partial t}=-\frac{1}{2 \hbar}\left(\rho_{22}-\rho_{11}\right)\left|\mu_{s}\right|^{2} \mathbf{E}_{s}^{\prime} \cdot \mathbf{e}_{s}-\frac{\eta_{s}^{\prime}}{T_{s}}$

$\frac{\partial \mathbf{E}_{s}^{\prime}}{\partial z}+\frac{1}{v} \frac{\partial \mathbf{E}_{s}^{\prime}}{\partial t}+\frac{\gamma_{s}}{2} \mathbf{E}_{\mathbf{s}}^{\prime}=-\frac{\mu \omega_{s}^{2}}{k_{s}} \int_{\Omega} n_{s}(\theta, \phi) \eta_{s}^{\prime} \mathbf{e}_{s} d \Omega$

where $\rho_{22}$ and $\rho_{11}$ are density matrix elements representing the populations of the upper and lower laser levels, and $\left|\mu_{\mathrm{s}}\right|$ and $\left|\mu_{\mathrm{p}}\right|$ are the magnitudes of the signal and pump dipole moments, respectively. These moments are defined as

$\mu_{\mathrm{p}}=\mu_{03}=\left|\mu_{p}\right| \mathbf{e}_{\mathrm{p}}$,

$\mu_{s}=\mu_{12}=\left|\mu_{p}\right| \mathbf{e}_{s}$.

The electromagnetic fields and the off-diagonal elements of the density matrix are assumed to be dominated by a plane wave form, which is propagating either longitudinally or transversely with respect to the longitudinal axis of the laser cavity. The parameter $\eta_{s}(\theta, \phi, z, t)$ is related to the slowly varying amplitude of the off-diagonal elements associated with the signal transition by $\eta_{s}^{\prime}=-i \rho_{21}\left|\mu_{s}\right|$, where $i$ is the imaginary number unit, $\rho_{21}$ is the off-diagonal element of the density matrix. $\mathrm{E}_{\mathrm{p}}^{\prime}(z, t)$ and $\mathrm{E}_{\mathrm{s}}^{\prime}(z, t)$ are the slowly varying amplitudes of the pump and signal fields. The variable $\theta$ measures the angle of a class of signal dipoles with respect to the $x$ axis (which is perpendicular to the direction of the signal propagation), and $\phi$ measures the orientation of the dipoles around the $x$ axis. The function $n_{s}(\theta, \phi) d \Omega$ represents the number of molecules per unit volume having their signal dipoles oriented within the solid angle $d \Omega$ about the $(\theta, \phi)$ direction. For the cases of interest here, the pump and signal dipoles of the dye molecules are parallel $\left(\mathbf{e}_{\mathbf{p}}=\mathbf{e}_{\mathbf{s}}\right)$ [27]. Even though Eqs (1)-(4) are a set of simplified Maxwell-densitymatrix equations for short-pulse dye lasers, they are still expressed in terms of 
fundamental physical quantities. Further modifications of this set of equations are necessary in order to make them useful for practical laser systems.

\section{Longitudinal Pumping}

It is assumed now that the signal field $\mathbf{E}^{\prime}$, is parallel to the $x$ axis, which is perpendicular to the signal pulse propagation direction, and $\mathbf{E}_{\mathbf{p}}^{\mathbf{p}}$ is linearly polarized in the $x y$ plane and oriented at an angle $\alpha$ with respect to the $x$ axis as shown in Figure 2. Then the electric field vectors and the unit dipole moment vectors can be written

$\mathbf{E}_{\mathrm{p}}^{\prime}=E_{p}^{\prime}\left(\mathbf{e}_{\mathrm{x}} \cos \alpha+\mathbf{e}_{\mathrm{y}} \sin \alpha\right)$,

$\mathbf{E}_{\mathbf{s}}^{\prime}=E_{s}^{\prime} \mathbf{e}_{\mathbf{x}}$,

$\mathbf{e}_{\mathrm{p}}=\mathbf{e}_{\mathrm{s}}=\mathbf{e}_{\mathrm{x}} \cos \theta+\mathbf{e}_{\mathrm{y}} \sin \theta \cos \phi+\mathbf{e}_{\mathrm{z}} \sin \theta \sin \phi$.

The dot products of these vectors in Eqs. (1)-(3) are

$$
\begin{aligned}
\mathbf{E}_{\mathbf{p}}^{\prime} \cdot \mathbf{e}_{\mathbf{p}} & =E_{p}^{\prime}\left(\mathbf{e}_{\mathbf{x}} \cos \alpha+\mathbf{e}_{\mathrm{y}} \sin \alpha\right) \cdot\left(\mathbf{e}_{\mathrm{x}} \cos \theta+\mathbf{e}_{\mathrm{y}} \sin \theta \cos \phi+\mathbf{e}_{\mathrm{z}} \sin \theta \sin \phi\right) \\
& =E_{p}^{\prime}(\cos \alpha \cos \theta+\sin \alpha \sin \theta \cos \phi) \\
& =E_{p}^{\prime}\left[\chi \cos \alpha+\left(1-\chi^{2}\right)^{1 / 2} \sin \alpha \cos \phi\right] \\
& =E_{p}^{\prime} X, \\
\mathbf{E}_{\mathbf{s}}^{\prime} \cdot \mathbf{e}_{\mathbf{s}} & =E_{s}^{\prime} \mathbf{e}_{\mathrm{x}} \cdot\left(\mathbf{e}_{\mathrm{x}} \cos \theta+\mathbf{e}_{\mathrm{y}} \sin \theta \cos \phi+\mathbf{e}_{\mathrm{z}} \sin \theta \sin \phi\right) \\
& =E_{s}^{\prime} \chi,
\end{aligned}
$$

where the new angle variables are

$\chi=\cos \theta$,

$X=\chi \cos \alpha+\left(1-\chi^{2}\right)^{1 / 2} \sin \alpha \cos \phi$.

It is evident that when the pump field is parallel to the signal field $(\alpha=0)$ the variable $X$ equals $\chi$. 


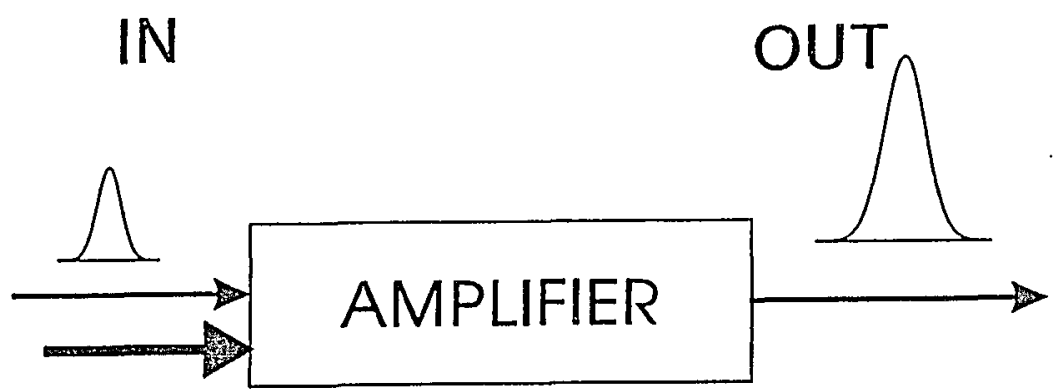

PUMP

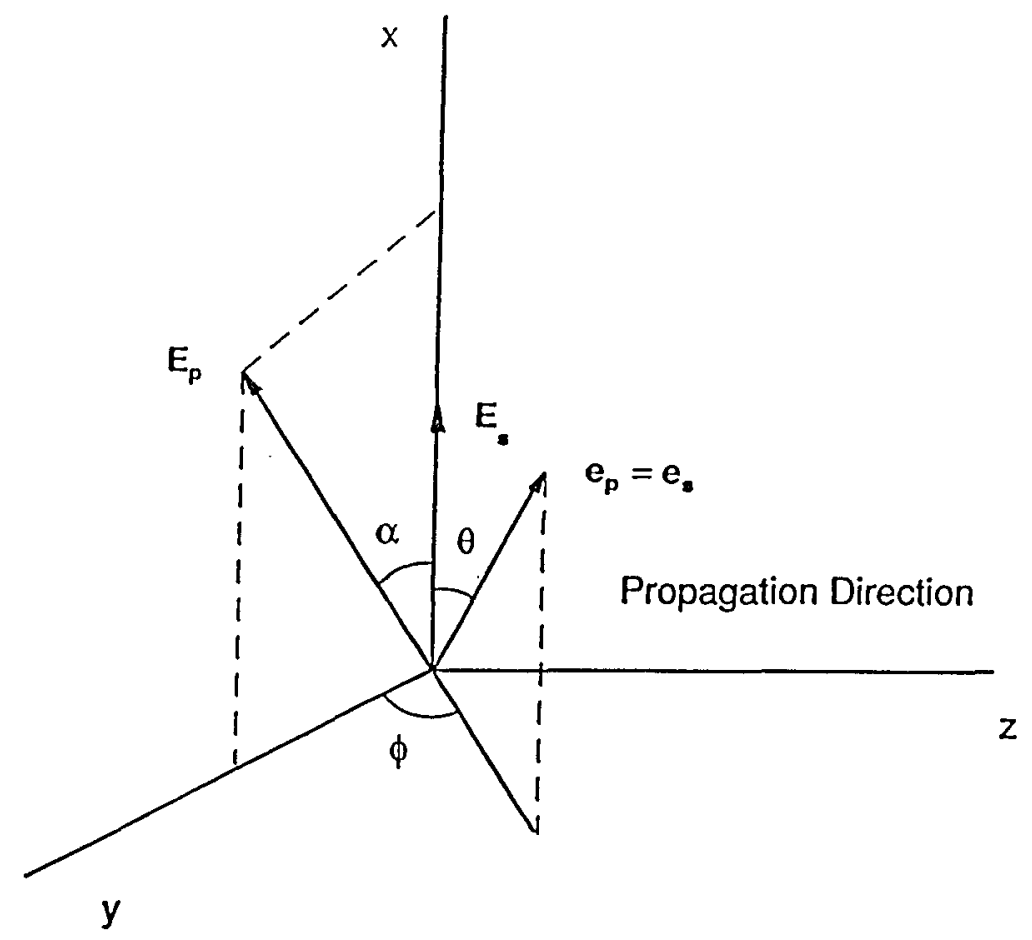

Figure 2. Geometry of pump and signal fields and molecular dipole moments in the longitudinal pump arrangement. 
With the substitutions given in Eqs. (10)-(13), Eqs. (1)-(3) become

$$
\begin{aligned}
& \frac{\partial \rho_{22}}{\partial t}=\frac{1}{\hbar} \eta_{s}^{\prime} E_{s}^{\prime} \chi-\frac{\rho_{22}}{\tau_{2}}+\frac{T_{p}\left|\mu_{p}\right|^{2}}{2 \hbar^{2}}\left(E_{p}^{\prime} X\right)^{2}, \\
& \frac{\partial \rho_{11}}{\partial t}=-\frac{1}{\hbar} \eta_{s}^{\prime} E_{s}^{\prime} \chi-\frac{\rho_{11}}{\tau_{1}}+\frac{\rho_{22}}{\tau_{2}}, \\
& \frac{\partial \eta_{s}^{\prime}}{\partial t}=-\frac{1}{2 \hbar}\left(\rho_{22}-\rho_{11}\right)\left|\mu_{s}\right|^{2} E_{s}^{\prime} \chi-\frac{\eta_{s}^{\prime}}{T_{s}} .
\end{aligned}
$$

For dye molecules in a laser amplifier having an isotropic orientational distribution, the angular distribution function can be written as $n_{s}(\theta, \phi)=N / 4 \pi$, where $N$ is the dye molecule concentration, and Eq. (4) reduces to

$$
\frac{\partial \mathbf{E}_{s}^{\prime}}{\partial z}+\frac{1}{v} \frac{\partial \mathbf{E}_{s}^{\prime}}{\partial t}+\frac{\gamma_{s}}{2} \mathbf{E}_{s}^{\prime}=-\frac{\mu \omega_{s}^{2}}{k_{s}} \frac{N}{2 \pi} \int_{0}^{1} \int_{0}^{2 \pi} \eta_{s}^{\prime} \chi d \chi d \phi
$$

By introducing suitably normalized forms for dependent variables, Eqs. (14)-(17) can be written in a more compact form. The normalization forms chosen here are the following:

$$
\begin{aligned}
& D(\theta, z, t)=\frac{\mu \omega_{s}^{2} N T_{s}\left|\mu_{s}\right|^{2}}{k_{s} \hbar \gamma_{s}} \frac{\chi^{2}\left(2-3 \sin ^{2} \alpha\right)+\sin ^{2} \alpha}{2 X^{2}}\left(\rho_{22}-\rho_{11}\right), \\
& M(\theta, z, t)=\frac{\mu \omega_{s}^{2} N T_{s}\left|\mu_{s}\right|^{2}}{k_{s} \hbar \gamma_{s}} \frac{\chi^{2}\left(2-3 \sin ^{2} \alpha\right)+\sin ^{2} \alpha}{2 X^{2}}\left(\rho_{22}+\rho_{11}\right), \\
& Q(\theta, z, t)=-\frac{\mu \omega_{s}^{2} N T_{s}\left|\mu_{s}\right|^{2}\left(2 \tau_{2} T_{s}\right)^{1 / 2}}{k_{s} \hbar \gamma_{s}} \frac{\chi^{2}\left(2-3 \sin ^{2} \alpha\right)+\sin ^{2} \alpha}{2 X^{2}} \eta_{s}^{\prime}, \\
& A(z, t)=\frac{\left|\mu_{s}\right|}{\hbar}\left(\frac{\tau_{2} T}{2}\right)^{1 / 2}\left|E_{s}^{\prime}\right|,
\end{aligned}
$$


$P(z, t)=\frac{\mu \omega_{s}^{2} N T_{s}\left|\mu_{s}\right|^{2}}{k_{s} \hbar \gamma_{s}} \frac{\tau_{2} T_{p}\left|\mu_{p}\right|^{2}\left|\mathbf{E}_{\mathrm{p}}^{\prime}\right|^{2}}{2 \hbar^{2}}$

where the fundamental independent variables are also noted on the left-hand side. Thus $D$ is a normalized population difference, $M$ is a normalized population sum, $Q$ is a normalized polarization, $A$ is a normalized signal electric field, and $P$ is a normalized pump rate.

In terms of the new variables given in Eqs. (18)-(22), Eqs. (14)-(17) simplify to

$\frac{\partial D}{\partial t}=-\frac{1}{\tau_{2}}\left\{\left(1+\frac{\tau_{2}}{2 \tau_{1}}\right) D+\left(1-\frac{\tau_{2}}{2 \tau_{1}}\right) M+2 Q A \chi-P\left[\chi^{2}\left(1-\frac{3 \sin ^{2} \alpha}{2}\right)+\frac{\sin ^{2} \alpha}{2}\right]\right\}$,

$\frac{\partial M}{\partial t}=-\frac{1}{\tau_{2}}\left\{-\frac{\tau_{2}}{2 \tau_{1}} D+\frac{\tau_{2}}{2 \tau_{1}} M-P\left[\chi^{2}\left(1-\frac{3 \sin ^{2} \alpha}{2}\right)+\frac{\sin ^{2} \alpha}{2}\right]\right\}$,

$\frac{\partial Q}{\partial t}=-\frac{1}{T_{s}}(Q-A D \chi)$

$\frac{\partial A}{\partial z}+\frac{1}{v} \frac{\partial A}{\partial t}=-\frac{\gamma_{s}}{2}\left(A-\int_{0}^{1} Q \chi d \chi\right)$

With this change of parameters all dependence on the $\phi$ variable is eliminated, and the pump misalignment is equivalent to a reduction of the pump amplitude.

\section{Transverse Pumping}

For the transverse pump arrangement as shown in Figure 3, the signal field $\mathbf{E}_{\mathbf{s}}{ }^{\prime}$ is parallel to the $x$ axis, and the pump field $\mathbf{E}_{\mathbf{p}}^{\prime}$ is linearly polarized in the $x z$ plane and oriented at an angle $\alpha$ with respect to the $x$ axis. Then the electric field vectors and the unit dipole moment vectors can be written

$\mathbf{E}_{\mathrm{p}}^{\prime}=E_{p}^{\prime}\left(\mathbf{e}_{\mathrm{x}} \cos \alpha+\mathbf{e}_{\mathrm{z}} \sin \alpha\right)$, 

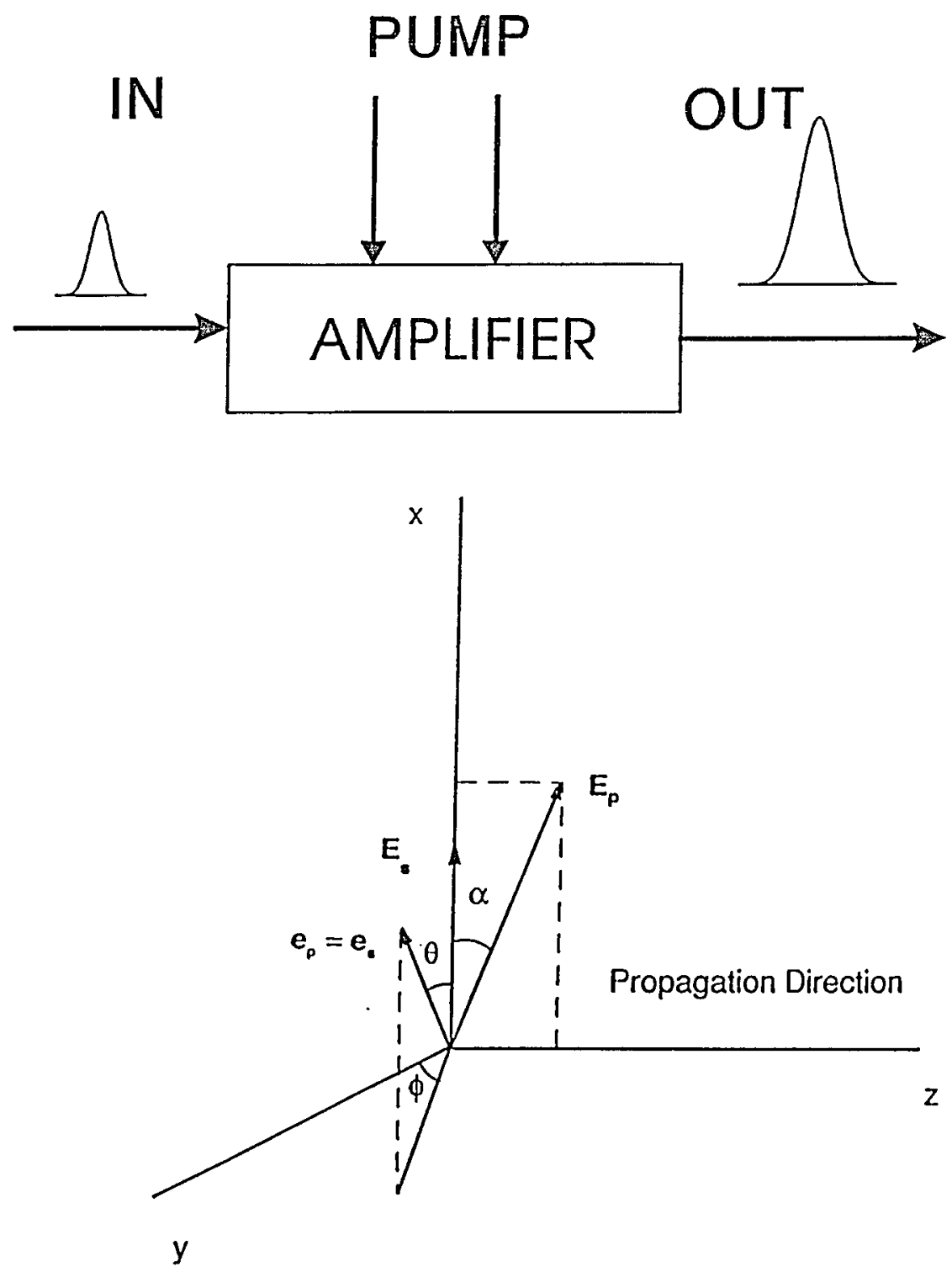

Figure 3. Geometry of pump and signal fields and molecular dipole moments in the transverse pump arrangement. 
$\mathbf{E}_{\mathrm{s}}^{\prime}=E_{s}^{\prime} \mathbf{e}_{\mathbf{x}}$,

$\mathbf{e}_{\mathrm{p}}=\mathbf{e}_{\mathrm{s}}=\mathbf{e}_{\mathrm{x}} \cos \theta+\mathbf{e}_{\mathrm{y}} \sin \theta \cos \phi+\mathbf{e}_{\mathbf{z}} \sin \theta \sin \phi$

The dot products of these vectors in Eqs. (1)-(3) are

$$
\begin{aligned}
\mathbf{E}_{\mathbf{p}}^{\prime} \cdot \mathbf{e}_{\mathrm{p}} & =E_{p}^{\prime}\left(\mathbf{e}_{\mathrm{x}} \cos \alpha+\mathbf{e}_{\mathrm{z}} \sin \alpha\right) \cdot\left(\mathbf{e}_{\mathrm{x}} \cos \theta+\mathbf{e}_{\mathrm{y}} \sin \theta \cos \phi+\mathbf{e}_{\mathrm{z}} \sin \theta \sin \phi\right) \\
& =E_{p}^{\prime}(\cos \alpha \cos \theta+\sin \alpha \sin \theta \sin \phi) \\
& =E_{p}^{\prime}\left[\chi \cos \alpha+\left(1-\chi^{2}\right)^{1 / 2} \sin \alpha \sin \phi\right] \\
& =E_{p}^{\prime} X, \\
\mathbf{E}_{\mathbf{s}}^{\prime} \cdot \mathbf{e}_{\mathbf{s}} & =E_{s}^{\prime} \mathbf{e}_{\mathbf{x}} \cdot\left(\mathbf{e}_{\mathrm{x}} \cos \theta+\mathbf{e}_{\mathrm{y}} \sin \theta \cos \phi+\mathbf{e}_{\mathbf{z}} \sin \theta \sin \phi\right) \\
& =E_{s}^{\prime} \chi,
\end{aligned}
$$

where $\chi$ has the same form as in Eq. (12) but $\mathrm{X}$ takes the form

$X=\chi \cos \alpha+\left(1-\chi^{2}\right)^{1 / 2} \sin \alpha \sin \phi$.

With the same procedures as in the longitudinal pump arrangement mentioned above, and the same normalization form as Eqs. (18)-(22) except that the pump orientation factor $\mathrm{X}$ takes form of Eq. (32) instead of Eq. (13), one obtains the same differential equations for $D, M$, and $Q$ as given in Eqs. (23)-(25). Thus, the only difference between longitudinal pump and transverse pump is the pump orientation factor $\mathrm{X}$ which is now normalized away for these three variables.

$$
\begin{aligned}
& \frac{\partial D}{\partial t}=-\frac{1}{\tau_{2}}\left\{\left(1+\frac{\tau_{2}}{2 \tau_{1}}\right) D+\left(1-\frac{\tau_{2}}{2 \tau_{1}}\right) M+2 Q A \chi-P\left[\chi^{2}\left(1-\frac{3 \sin ^{2} \alpha}{2}\right)+\frac{\sin ^{2} \alpha}{2}\right]\right\}, \\
& \frac{\partial M}{\partial t}=-\frac{1}{\tau_{2}}\left\{-\frac{\tau_{2}}{2 \tau_{1}} D+\frac{\tau_{2}}{2 \tau_{1}} M-P\left[\chi^{2}\left(1-\frac{3 \sin ^{2} \alpha}{2}\right)+\frac{\sin ^{2} \alpha}{2}\right]\right\}, \\
& \frac{\partial Q}{\partial t}=-\frac{1}{T_{s}}(Q-A D \chi),
\end{aligned}
$$


By substituting $\mathbf{E}_{\mathrm{s}}^{\prime}$ in terms of $A$, and $\eta_{\mathrm{s}}^{\prime}$ in terms of $Q$ into Eq. (4) and canceling all the common terms on both sides, one can obtain

$$
\frac{\partial A}{\partial z}+\frac{1}{v} \frac{\partial A}{\partial t}=-\frac{\gamma_{s}}{2} A+\frac{\gamma_{s}}{4 \pi} \int_{0}^{1} \int_{0}^{2 \pi} Q \chi \frac{\mathrm{X}^{2}}{\chi^{2}\left(1-\frac{3}{2} \sin ^{2} \alpha\right)+\frac{\sin ^{2} \alpha}{2}} d \phi d \chi
$$

Substituting Eq. (32) into Eq. (36), and carrying out the integral over $\phi$, one obtains

$$
\begin{aligned}
\frac{\partial A}{\partial z}+\frac{1}{v} \frac{\partial A}{\partial t} & =-\frac{\gamma_{s}}{2} A+\frac{\gamma_{s}}{4 \pi} \int_{0}^{1} \int_{0}^{2 \pi} Q \chi \frac{\left[\chi \cos \alpha+\left(1-\chi^{2}\right)^{1 / 2} \sin \alpha \sin \phi\right]^{2}}{\chi^{2}\left(1-\frac{3}{2} \sin ^{2} \alpha\right)+\frac{\sin ^{2} \alpha}{2}} d \phi d \chi \\
& =-\frac{\gamma_{s}}{2} A+\frac{\gamma_{s}}{4 \pi} \int_{0}^{1} \int_{0}^{2 \pi} Q \chi \frac{\chi^{2} \cos ^{2} \alpha+\left(1-\chi^{2}\right) \sin ^{2} \alpha \sin ^{2} \phi+2 \chi \cos \alpha\left(1-\chi^{2}\right)^{1 / 2} \sin \alpha \sin \phi}{\chi^{2}\left(1-\frac{3}{2} \sin ^{2} \alpha\right)+\frac{\sin ^{2} \alpha}{2}} d \phi d \chi \\
& =-\frac{\gamma_{s}}{2} A+\frac{\gamma_{s}}{4 \pi} \int_{0}^{1} \frac{Q \chi}{\chi^{2}\left(1-\frac{3}{2} \sin ^{2} \alpha\right)+\frac{\sin ^{2} \alpha}{2}} 2 \pi\left[\chi^{2} \cos ^{2} \alpha+\frac{\left(1-\chi^{2}\right) \sin ^{2} \alpha}{2}\right] d \chi \\
& =-\frac{\gamma_{s}}{2} A+\frac{\gamma_{s}}{2} \int_{0}^{1} \frac{Q_{\chi}}{\chi^{2}\left(1-\frac{3}{2} \sin ^{2} \alpha\right)+\frac{\sin ^{2} \alpha}{2}}\left[\chi^{2}\left(1-\sin ^{2} \alpha\right)+\frac{\left(1-\chi^{2}\right) \sin ^{2} \alpha}{2}\right] d \chi \\
& =-\frac{\gamma_{s}}{2}\left(A-\int_{0}^{1} Q \chi d \chi\right),
\end{aligned}
$$

which is the same as Eq. (26). Thus, Eqs. (23)-(26) can be used for both longitudinal and transverse pump arrangements, and may be used to analyze light-matter interaction in a dye laser amplifier having arbitrary space- and time-dependent pump and loss rates.

\section{Rate Equation Approximation}

In the rate equation approximation, one ignores coherence effects and therefore the dependence of the polarization on past values of the electric field. Since a finite coherence time represents a temporal lag between the induced polarization and the electric field, coherence effects play an important role in limiting the magnitude and width of 
optical pulses $[16,17,19,23,28,29]$. However, when the optical pulse width is long compared to the coherence time, the rate equation approximation (or zero coherence time approximation $T_{s}=0$ ) is often used. In this approximation, the polarization varies simultaneously with the electric field. As a result, Eq.(25) has the solution

$Q=A D \chi$.

With this substitution, Eqs. (23)-(24) and (26) become

$$
\begin{aligned}
& \frac{\partial D}{\partial t}=-\frac{1}{\tau_{2}}\left\{\left(1+\frac{\tau_{2}}{2 \tau_{1}}\right) D+\left(1-\frac{\tau_{2}}{2 \tau_{1}}\right) M+2 A^{2} D \chi^{2}-P\left[\chi^{2}\left(1-\frac{3 \sin ^{2} \alpha}{2}\right)+\frac{\sin ^{2} \alpha}{2}\right]\right\} \\
& \frac{\partial M}{\partial t}=-\frac{1}{\tau_{2}}\left\{-\frac{\tau_{2}}{2 \tau_{1}} D+\frac{\tau_{2}}{2 \tau_{1}} M-P\left[\chi^{2}\left(1-\frac{3 \sin ^{2} \alpha}{2}\right)+\frac{\sin ^{2} \alpha}{2}\right]\right\} \\
& \frac{\partial A}{\partial z}+\frac{1}{v} \frac{\partial A}{\partial t}=-\frac{\gamma_{s}}{2} A\left(1-\int_{0}^{1} D \chi^{2} d \chi\right)
\end{aligned}
$$

By defining the intensity $I \equiv A^{2}$, we can write Eqs. (39)-(41) in the following form in terms of the normalized population difference $D$, population $\operatorname{sum} M$, and intensity $I$ :

$$
\begin{aligned}
& \frac{\partial D}{\partial t}=-\frac{1}{\tau_{2}}\left\{\left(1+\frac{\tau_{2}}{2 \tau_{1}}\right) D+\left(1-\frac{\tau_{2}}{2 \tau_{1}}\right) M+2 I D \chi^{2}-P\left[\chi^{2}\left(1-\frac{3 \sin ^{2} \alpha}{2}\right)+\frac{\sin ^{2} \alpha}{2}\right]\right\}, \\
& \frac{\partial M}{\partial t}=-\frac{1}{\tau_{2}}\left\{-\frac{\tau_{2}}{2 \tau_{1}} D+\frac{\tau_{2}}{2 \tau_{1}} M-P\left[\chi^{2}\left(1-\frac{3 \sin ^{2} \alpha}{2}\right)+\frac{\sin ^{2} \alpha}{2}\right]\right\} \\
& \frac{\partial I}{\partial z}+\frac{1}{v} \frac{\partial I}{\partial t}=-\gamma_{s} I\left(1-\int_{0}^{1} D \chi^{2} d \chi\right) .
\end{aligned}
$$

In this limit, a set of three nonlinear coupled differential equations, instead of four as in the above sections, is employed to describe the light-matter interaction in a dye laser amplifier. It is also noted that this set deals with pulse intensity instead of with electric 
field. Thus, the rate equation model can not explain the polarization effects of pump and signal fields.

\section{Unidirectional Molecular Distribution Model}

For quantitative investigation of ultrashort dye laser amplifiers, the isotropic molecular distribution is required as in the previous subsections above. With parallel pump and signal polarizations and modest pump power, however, the unidirectional molecular distribution is a common way to simplify and qualitatively describe the interactions in dye lasers as well as to use less computer time.

A unidirectional orientational distribution can be written as $n(\theta, \phi)=N \delta\left(\theta-\theta_{0}, \phi-\phi_{0}\right)$, where $\delta$ is a normalized Dirac-delta function, and $\theta_{0}$ and $\phi_{0}$ indicate the alignment direction. If this distribution is substituted into Eqs. (14)-(17) with $\theta_{0}=0$, and $\alpha=0$, and proceeding as before, one finds that the final set of Eqs.(23)-(26) are replaced by the unidirectional set

$$
\begin{aligned}
& \frac{\partial D}{\partial t}=-\frac{1}{\tau_{2}}\left\{\left(1+\frac{\tau_{2}}{2 \tau_{1}}\right) D+\left(1-\frac{\tau_{2}}{2 \tau_{1}}\right) M+2 Q A-P\right\}, \\
& \frac{\partial M}{\partial t}=-\frac{1}{\tau_{2}}\left\{-\frac{\tau_{2}}{2 \tau_{1}} D+\frac{\tau_{2}}{2 \tau_{1}} M-P\right\}, \\
& \frac{\partial Q}{\partial t}=-\frac{1}{T_{s}}(Q-A D), \\
& \frac{\partial A}{\partial z}+\frac{1}{v} \frac{\partial A}{\partial t}=-\frac{\gamma_{s}}{2}(A-Q) .
\end{aligned}
$$

\section{THEORY OF SYNCHRONOUSLY PUMPED MODE-LOCKED DYE LASERS}

When periodic picosecond pulses are required, a standard technique involves synchronous pumping of the dye laser medium using as the pump source a mode-locked 
argon laser or a frequency-doubled mode-locked Nd:YAG laser [7, 8, 16, 25]. Typically, the pump laser is mode-locked acoustically, and the length of the dye laser cavity is adjusted to be almost exactly equal to the pump laser cavity length (or a multiple thereof). In operation, the dye laser pulse arrives at the dye jet approximately simultaneously with the pump pulse, and this arrangement is found to favor mode-locking of the dye laser.

In a synchronously pumped mode-locked dye laser, as mentioned above, the signal field amplitude $A$ must be periodic with the same period as the pump pulse. If the net gain per pass in the laser amplifier is not too large, each molecule in the dye medium interacts with the same time-dependent pump and signal amplitudes. Thus, the output of the dye laser is the same as it would be if the gain and loss were uniformly distributed around the dye cavity with the pump and signal pulses having exactly equal velocity. There is, however, no reason why these velocities should be considered to be equal to the speed of light $v$. The repetition rate of the pump pulse is governed primarily by parameters of the pump laser, and with saturable gain, it is possible for the effective velocity of the dye laser signal to be somewhat greater than or less than the speed of light. To take advantage of these concepts, it is helpful to introduce a new time coordinate $\tau=t-z / v_{s}$, where $v_{s}$ is the "envelope speed" in the dye laser of all quantities of interest. Then Eqs. (23)-(26) reduce to the ordinary differential equations

$$
\begin{aligned}
& \frac{d D}{d \tau}=-\frac{1}{\tau_{2}}\left\{\left(1+\frac{\tau_{2}}{2 \tau_{1}}\right) D+\left(1-\frac{\tau_{2}}{2 \tau_{1}}\right) M+2 Q A \chi-P\left[\chi^{2}\left(1-\frac{3 \sin ^{2} \alpha}{2}\right)+\frac{\sin ^{2} \alpha}{2}\right]\right\}, \\
& \frac{d M}{d \tau}=-\frac{1}{\tau_{2}}\left\{-\frac{\tau_{2}}{2 \tau_{1}} D+\frac{\tau_{2}}{2 \tau_{1}} M-P\left[\chi^{2}\left(1-\frac{3 \sin ^{2} \alpha}{2}\right)+\frac{\sin ^{2} \alpha}{2}\right]\right\}, \\
& \frac{d Q}{d \tau}=-\frac{1}{T_{s}}(Q-A D \chi),
\end{aligned}
$$


$\frac{d A}{d \tau}=-\frac{1}{2 t_{c}\left(1-v / \nu_{s}\right)}\left(A-\int_{0}^{1} Q \chi d \chi\right)$,

where all of the cavity losses are now incorporated in the photon-cavity lifetime.

As one of the most important measurable parameters in a study of synchronous mode-locking is the relative length of the dye laser cavity, it is convenient in Eq.(52) to express the velocities in terms of the lengths of the pump and dye laser cavities. Thus, in terms of the cavity length mismatch, or the cavity length difference $\Delta L$, and the dye laser cavity length $L$, Eq. (52) can be written [16]

$\frac{d A}{d \tau}=-\frac{L}{2 t_{c} \Delta L}\left(A-\int_{0}^{1} Q \chi d \chi\right)$

Eqs.(49)-(51) and (53) are the key equations for the semiclassical treatment for a synchronously pumped mode-locked dye laser including finite coherence time and isotropic molecular distribution.

For the rate equation approximation, as discussed for the laser amplifier, a set of equations in this limit can be obtained as

$$
\begin{aligned}
& \frac{d D}{d \tau}=-\frac{1}{\tau_{2}}\left\{\left(1+\frac{\tau_{2}}{2 \tau_{1}}\right) D+\left(1-\frac{\tau_{2}}{2 \tau_{1}}\right) M+2 I D \chi^{2}-P\left[\chi^{2}\left(1-\frac{3 \sin ^{2} \alpha}{2}\right)+\frac{\sin ^{2} \alpha}{2}\right]\right\} \\
& \frac{d M}{d \tau}=-\frac{1}{\tau_{2}}\left\{-\frac{\tau_{2}}{2 \tau_{1}} D+\frac{\tau_{2}}{2 \tau_{1}} M-P\left[\chi^{2}\left(1-\frac{3 \sin ^{2} \alpha}{2}\right)+\frac{\sin ^{2} \alpha}{2}\right]\right\} \\
& \frac{d I}{d \tau}=-\frac{L I}{t_{c} \Delta L}\left(1-\int_{0}^{1} D \chi^{2} d \chi\right)
\end{aligned}
$$

where $D, M$, and $I$ have the same meanings as introduced before.

Similarly, equations for the unidirectional molecular distribution can be written 


$$
\begin{aligned}
& \frac{d D}{d \tau}=-\frac{1}{\tau_{2}}\left\{\left(1+\frac{\tau_{2}}{2 \tau_{1}}\right) D+\left(1-\frac{\tau_{2}}{2 \tau_{1}}\right) M+2 Q A-P\right\}, \\
& \frac{d M}{d \tau}=-\frac{1}{\tau_{2}}\left\{-\frac{\tau_{2}}{2 \tau_{1}} D+\frac{\tau_{2}}{2 \tau_{1}} M-P\right\}, \\
& \frac{d Q}{d \tau}=-\frac{1}{T_{s}}(Q-A D), \\
& \frac{d A}{d \tau}=-\frac{L}{2 t_{c} \Delta L}(A-Q) .
\end{aligned}
$$

\section{CONCLUSION}

In this chapter, we have developed a general semiclassical model, Eqs. (23)-(26) for an ultrashort dye laser amplifier, and Eqs. (49)-(51) and (63) for a synchronously pumped mode-locked dye laser. This semiclassical model started from Maxwell's equations for the electric field and density matrix equations for the active laser medium. It includes a finite coherence time, or phase memory time of the molecular wave function, a finite vibrational relaxation time for the lower electronic state of the dye laser transition, and an isotropic molecular orientational distribution. As the model deals with the field vectors directly, it will allow one to investigate the pump and signal polarization effects. Moreover, a corresponding rate equation model, Eqs.(42)-(44) for amplifiers, and Eqs.(54)-(56) for oscillators was also developed. By comparing the results obtained from both a more general semiclassical model and a zero coherence time rate equation model, one can investigate the coherence effects concerning the pulse parameters, and also the validity and inaccuracies of the commonly used rate equation approximation. Finally, a unidirectional molecular distribution model, Eqs.(45)-(48) for amplifiers and Eqs. (57)(60) for oscillators, may also permit qualitative studies. In conclusion, models developed 
in this chapter will be the cornerstone for investigating dynamics in ultrashort pulsed dye laser amplifiers and oscillators. 


\section{CHAPTER III}

\section{PUMP POLARIZATION EFFECTS IN SYNCHRONOUSLY PUMPED MODE-LOCKED DYE LASERS}

\section{INTRODUCTION}

Lasers can operate with unpolarized pumping mechanisms such as flashlamps and electric discharges, or polarized pumping mechanisms such as other lasers. By introducing some anisotropic components into the resonator or lasing medium, all lasers can produce polarized output light. Examples include solid-state lasers, when the host crystal is biaxial or uniaxial, and gas lasers, when a Brewster window is used on the laser tube. Dye lasers, in particular, are often designed to use other lasers as their pump sources. The pump lasers are typically linearly polarized, which favors the gain for one signal polarization. Some studies have been carried out previously concerning the polarization characteristics of the spontaneous and stimulated emission from dye laser medium [27, 30-34]. Under conditions of $\mathrm{cw}$ or quasi-cw pumping the polarization characteristics of dye laser amplifiers have also been investigated [35-37]. Due to their unique properties of high efficiency, fast decay time, and wide frequency tuning range, dye lasers have made possible the production of tunable picosecond and subpicosecond pulses by means of mode-locking techniques $[7,10,38-40]$. When periodic picosecond pulses are required, a standard method involves the synchronous pumping of the dye medium with mode-locked argon, krypton, or frequency-doubled $\mathrm{Nd}^{3+} \mathrm{YAG}$ lasers as the pumping source. In practice, the length of the dye laser cavity is adjusted to be almost exactly equal to the pump laser cavity length (or a multiple thereof), so that the dye laser pulse arrives at the 
dye jet approximately simultaneously with the pump pulse, and such arrangement is found to favor the mode-locking of the dye laser [41-43].

Synchronously pumped mode-locked (SPML) dye lasers have been widely used as sources of tunable intense and stable picosecond and subpicosecond light pulses in the visible and infrared. These ultrashort optical pulses are used extensively in areas like highspeed time-resolved spectroscopy, fast electro-optic applications, etc. To date, there have been many theoretical analyses of the synchronously pumped mode-locking process $[9,11$, $13,14,16,44-46]$. Some of these analyses started from a rate equation model, and show that the cavity length of the dye laser and the pump laser should be matched very closely to obtain optimum performance $[9,11,44]$. While the rate equation analyses can qualitatively explain many aspects of the mode-locking process in SPML dye lasers, . semiclassical effects have been found to have a significant influence on the pulsation waveforms $[16,45,46]$.

Although many approaches have been employed in investigating ultrashort pulsation, none of these has addressed the effects of pump polarization in SPML dye lasers. As most SPML dye lasers are used to generate picosecond or subpicosecond pulses, the pump pulses usually have a pulse width on the order of hundred picoseconds, which is on the same order as the effective lifetime of the excited state. Therefore, the investigation of the polarization characteristics of SPML dye lasers based on the condition of $\mathrm{cw}$ or quasi-cw pumping [35-37] is probably not appropriate. The present work in this chapter includes detailed theoretical and experimental studies of pump polarization effects in SPML dye lasers. 
NUMERICAL RESULTS

As rate equation models deal with pulse intensity instead of field, one has to use the semiclassical model to study the pump polarization effects. The model used here is the one derived in Chapter II, Eqs. (49)-(51) and (53)

$$
\begin{aligned}
& \frac{\partial D}{\partial \tau}=-\frac{1}{\tau_{2}}\left\{\left(1+\frac{\tau_{2}}{2 \tau_{1}}\right) D+\left(1-\frac{\tau_{2}}{2 \tau_{1}}\right) M+2 Q A \chi-P\left[\chi^{2}\left(1-\frac{3 \sin ^{2} \alpha}{2}\right)+\frac{\sin ^{2} \alpha}{2}\right]\right\} \\
& \frac{\partial M}{\partial \tau}=-\frac{1}{\tau_{2}}\left\{-\frac{\tau_{2}}{2 \tau_{1}} D+\frac{\tau_{2}}{2 \tau_{1}} M-P\left[\chi^{2}\left(1-\frac{3 \sin ^{2} \alpha}{2}\right)+\frac{\sin ^{2} \alpha}{2}\right]\right\} \\
& \frac{\partial Q}{\partial \tau}=-\frac{1}{T_{s}}(Q-A D \chi), \\
& \frac{\partial A}{\partial \tau}=-\frac{L}{2 t_{c} \Delta L}\left(A-\int_{0}^{1} Q \chi d \chi\right),
\end{aligned}
$$

where all quantities have the same meanings as before. One of the key variables here, however, is the polarization misalignment angle $\alpha$. This variable measures the polarization angle between the pump field and the signal field. As illustrated in Figures 2 and 3, the signal electric field $\boldsymbol{E}_{\mathrm{s}}$ is polarized in the $x$ direction and the pump filed $\boldsymbol{E}_{\mathrm{p}}$ is polarized in an arbitrary direction having the angle $\alpha$ with respect to the signal field polarization.

It is the purpose of this section to study the pulse parameters as functions of the pump and signal field polarization misalignment angle $\alpha$. Before numerically solving Eqs. (62)-(64), it is necessary to specify the pump function $P(\tau)$. By choosing a Gaussian profile to describe the pump pulse, the pump function appearing in the nioudel can be expressed as

$$
P(\tau)=P_{0}\left(\frac{2}{\Delta \tau_{p}}\right)\left(\frac{\ln 2}{\pi}\right)^{1 / 2} \exp \left[-\left(\frac{2 \tau}{\Delta \tau_{p}}\right)^{2} \ln 2\right]
$$


where $\Delta \tau_{p}$ is the full width at half maximum. The constant $P_{0}$ can be related to the laser's operating point with respect to the oscillation threshold. Instead of using $P_{0}$ directly, it is helpful to introduce an associated threshold parameter $r$. The relationship between $P_{0}$ and $r$ can be found from the model using the relationship

$$
r=\frac{\int_{0}^{1} Q \chi d \chi}{A}
$$

with the population depletion term $2 Q A \chi$ and the pump misalignment $\alpha$ set to zero in the population equations. Experimentally, one can determine the threshold parameter by measuring the ratio of the pump power under operating conditions to its value at the lasing threshold.

For the coefficients appearing in Eqs. (62)-(64), we have used the best available values for the Rhodamine $6 \mathrm{G}$ dye laser system. These values include the fluorescence decay time $\tau_{2}=5 \times 10^{-9} \mathrm{~s}$, the vibrational relaxation time $\tau_{1}=1 \times 10^{-12} \mathrm{~s}$, and the coherence time $T_{s}=5 \times 10^{144} \mathrm{~s}$. Other parameters that are specific to our synchronously pumped mode-locked dye laser experiments include the pump pulse width $\Delta \tau_{\mathrm{p}}=100 \times 10^{-12} \mathrm{~s}$, the cavity lifetime $t_{\mathrm{c}}=6 \times 10^{-9} \mathrm{~s}$, the cavity length $L=1.8 \mathrm{~m}$, and the cavity length mismatch $\Delta L$ $=2 \times 10^{-5} \mathrm{~m}$. Using these parameter values, the predictions of the model have been compared with experimental data.

The first pulse parameter of interest is the pulse temporal profile. Curves in Figure 4 show the theoretical normalized intensity traces $I=A^{2}$ for different polarization angles at a particular value of threshold parameter. Figures $4(a)-4(c)$ show this polarization dependency for the threshold parameter values $r=1.3,1.46$, and 4.0 , respectively. The change of the vertical scales between the figures should be noted. 

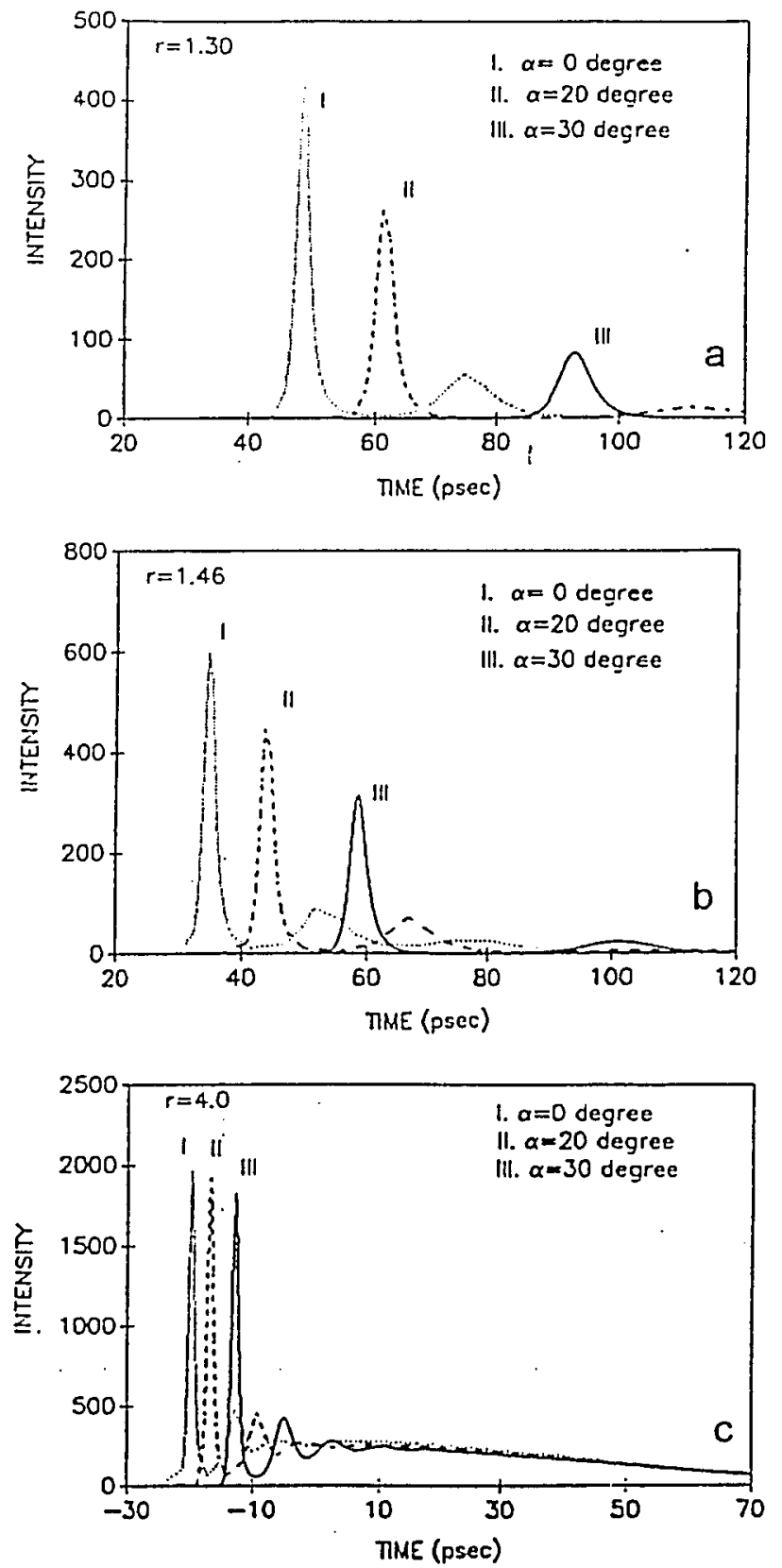

Figure 4. Temporal traces of the output pulses with pump polarization angles of $0^{\circ}, 20^{\circ}$, and $30^{\circ}$ for the threshold parameters (a) $r=1.3$, (b) 1.46 , and (c) 4.0. The different intensity scales should be noted. 
It is clear from these figures that with larger values of the threshold parameter and smaller values of the polarization angle the pulses become larger and earlier (relative to the peak of the pump pulse), and the ringing becomes faster. Because of the importance of the autocorrelation of the intensity pulses, we have also computed the autocorrelations of the pulses shown in Figure 4 using the formula

$G^{2}(\tau)=\int_{-\infty}^{+\infty} I\left(\tau^{\prime}\right) I\left(\tau+\tau^{\prime}\right) d \tau^{\prime}$

The results are shown in Figure 5. To aid in the comparison of pulse shapes, each autocorrelation curve is normalized by its maximum value.

For the dye laser pulses, the signal pulse width $\Delta \tau_{\mathrm{s}}$ is also an important parameter. Figure 6 gives curves of pulse width versus the pump polarization angles for several different values of the threshold parameters. For higher levels of pumping the pulses are shorter and less sensitive to the polarization angle. Also, with larger values of the threshold parameter, lasing still occurs with a $90^{\circ}$ rotation of the pump. This is because the pump polarization mostly favors the population inversion of the dipoles oriented approximately parallel to the field. When the pump becomes stronger, however, there are enough excited for the gain at these angles to also exceed the loss (even near the $90^{\circ}$ direction). Another parameter of interest is the peak intensity of the dye laser pulses $I_{p k}$. A set of curves of $I_{p k} v s . \alpha$ is shown in Figure 7.

In order to see the angular dependence more clearly, we have calculated the normalized average power of the pulses

$P_{a}=\frac{1}{T} \int_{0}^{T} S_{0} I(t) d t$,

where $S_{0}$ is the effective area at the dye laser output and $T$ is the period of the pulse train. As $T$ and $S_{o}$ are determined by the mode locker and the resonator geometry, they are 

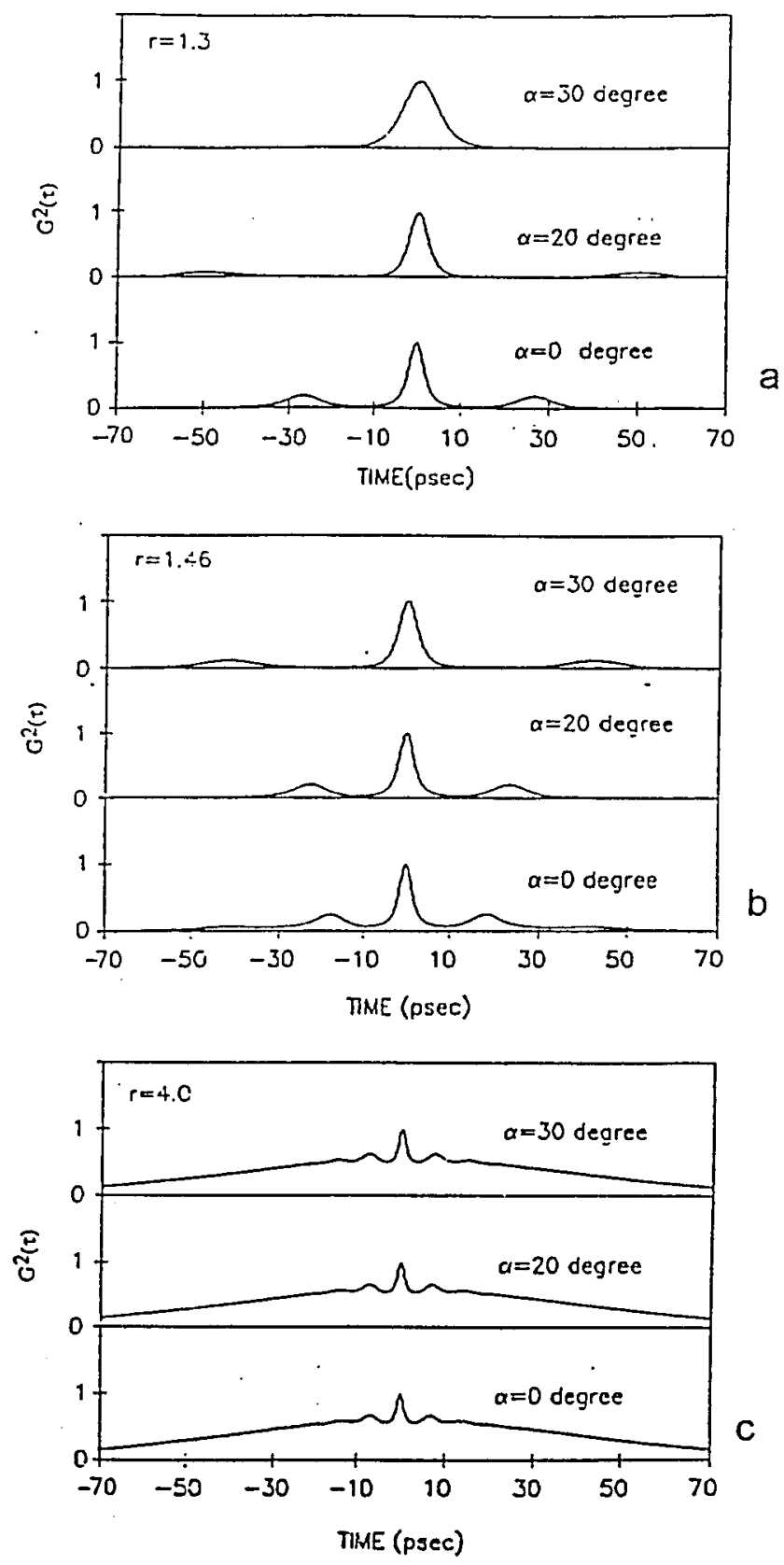

Figure 5. Intensity autocorrelations of the output pulses with pump polarization angles of $0^{\circ}, 20^{\circ}$, and $30^{\circ}$ for the threshold parameters (a) $r=$ 1.3 , (b) 1.46 , and (c) 4.0 . 


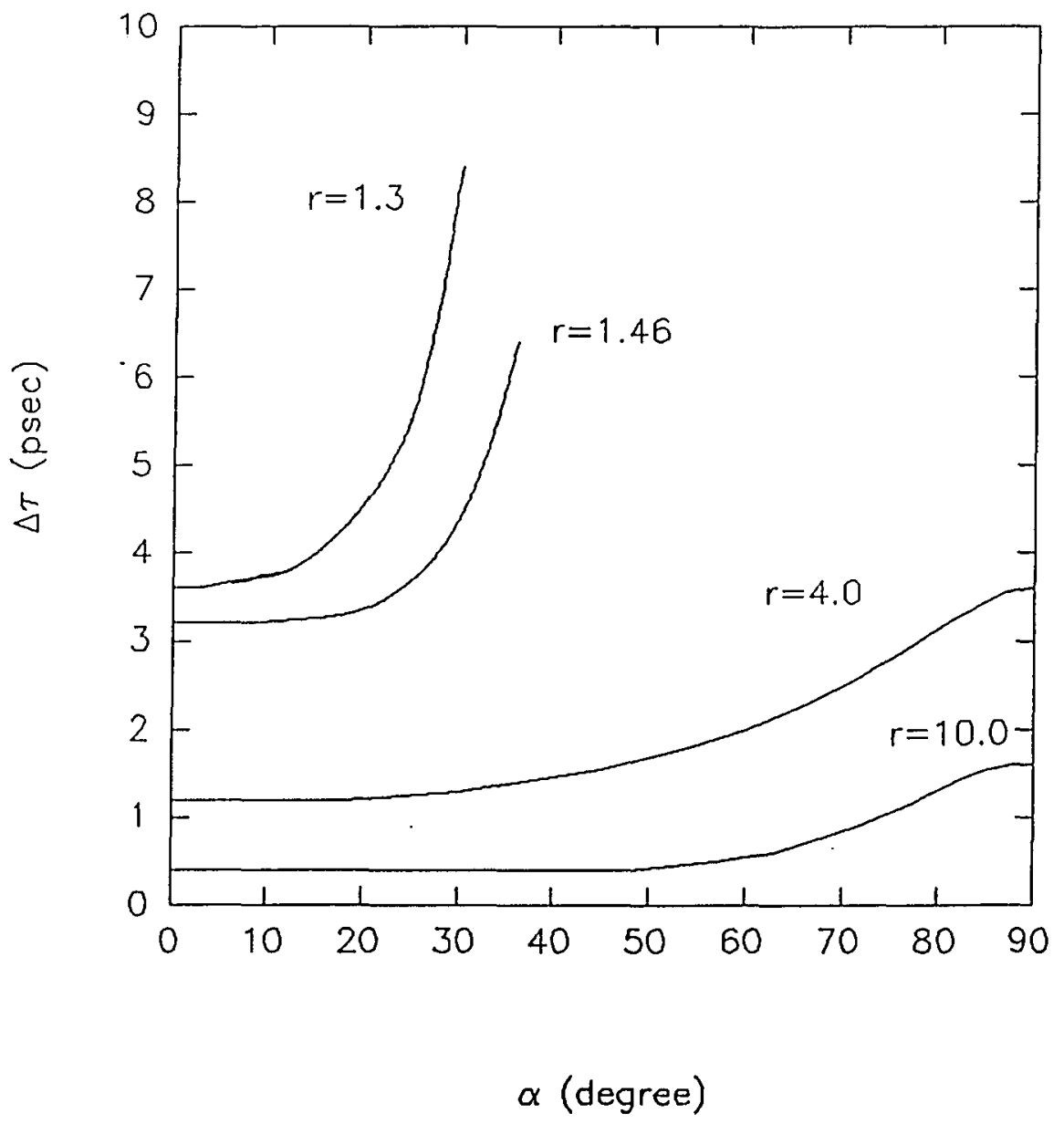

Figure 6. Theoretical pulse-width vs. the pump polarization angle for the threshold parameters $r=1.3,1.46,4.0$, and 10.0. 


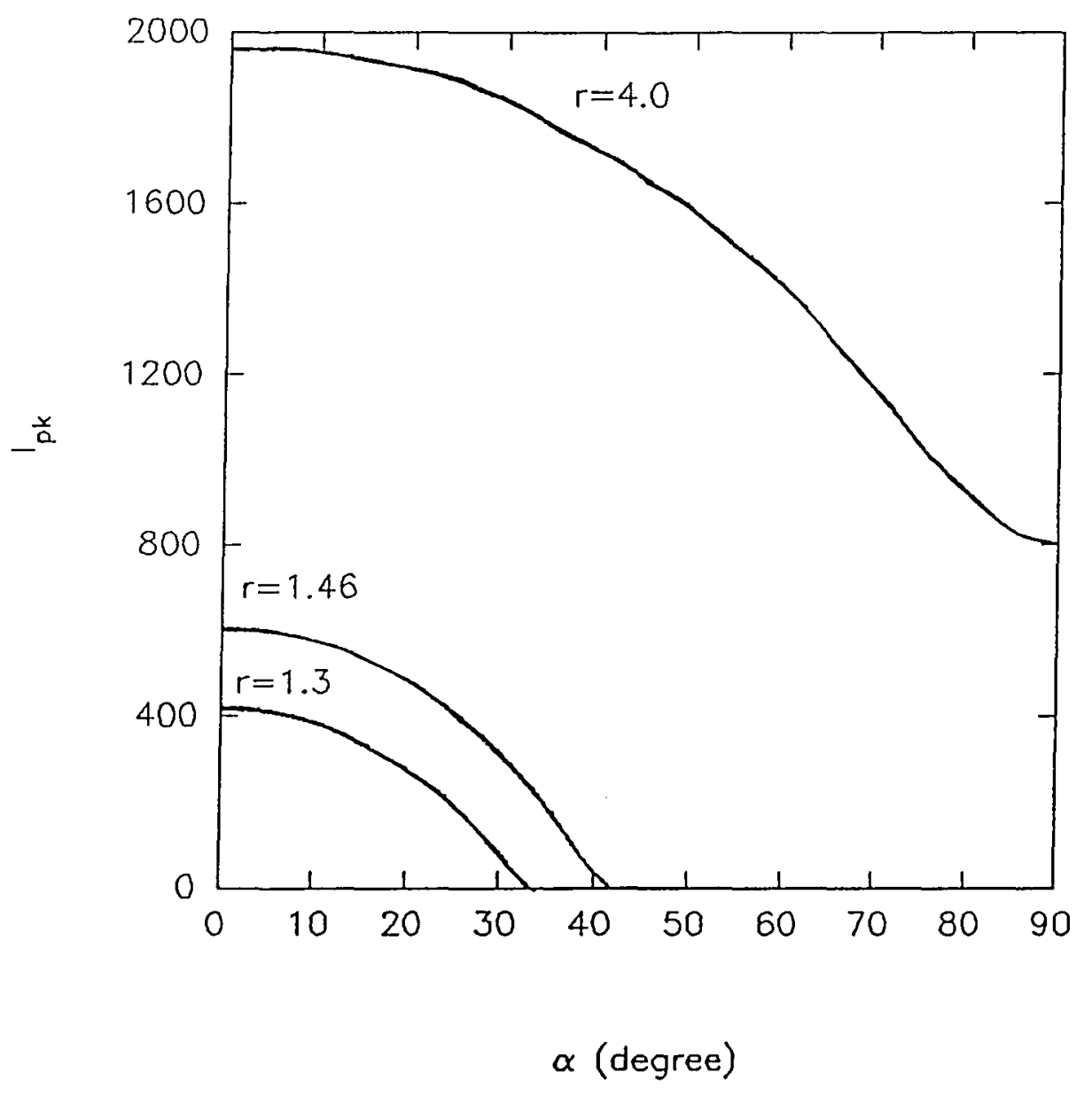

Figure 7. Output pulse peak intensities vs. pump polarization angle for the threshold parameters $r=1.3,1.46$, and 4.0. 
constant for one system. For each pump level, we have further normalized the average power for different polarization directions to the maximum average power with $\alpha=0$. In Figure 8 , the solid curves going from inside to outside correspond to the threshold parameter values $r=1.3,1.46,4.0$, and 10.0. If a vector is drawn from the origin to the point on the curve, the angle of the vector with respect to the $x$ axis represents the direction of the pump polarization, and the length of the vector represents the normalized average power for that polarization. The curves in Figure 9 reveal how the peak-to-peak time delay between the dye laser pulse and the pump pulse varies with change of pump polarization. As also noted previously in Figure 4, the pulse delay increases with pump misalignment.

\section{EXPERIMENTAL RESULTS}

The system used in the experiments involves an acousto-optically mode-locked Spectra-physics 2020 argon-ion laser as the pump source for a Spectra-Physics 375B dye laser, together making a synchronously pumped mode-locked dye laser system. The dye jet is set at Brewster's angle, so the dye laser electric field is forced to polarize in the $x$ direction, which is perpendicular to the pulse propagation direction. The pump polarization was controlled by a Newport polarization rotator which has a $2^{\circ}$ angular resolution. By inserting neutral density filters before the dye laser, the pump power could be adjusted. As the way mentioned above to determine the pump threshold parameter, we have chosen the threshold parameter value of $1.3,1.46$, and 2.83 for our experimental measurements. A Coherent power meter was used to measure the average output power. The experimental normalized average power versus the pump polarization angle is shown in Figure 8, where the open, filled squares and the open circles represent the pump levels of 1.3, 1.46 and 2.83, respectively. Using the methods described in Reference [47], the 
peak-to-peak time delay was also evaluated, and the results are shown in Figure 9 by the open and filled triangles.

\section{CONCLUSION}

With a linearly polarized pumping field, the pulsations of a synchronously pumped mode-locked dye laser have been shown to depend strongly on the pump polarization angle. This angular dependence is found to be less for large values of the threshold parameters. The linearly polarized pump field favors the population inversion of the dipoles oriented approximately parallel to the field. When the pump field is weak, those dipoles near the pump field direction will thus give the greatest contribution to the gain in the $x$ direction, so the gain for the signal field mostly depends on the contribution of the dipoles in the direction of the pump field. When the pump field becomes stronger, however, enough excited dipoles can make lasing possible in other directions even near the $90^{\circ}$ direction with respect to the signal field. The experimental pump polarization and pulse delay behavior of the synchronously pumped mode-locked dye laser are found to be in excellent agreement with the theoretical model. 


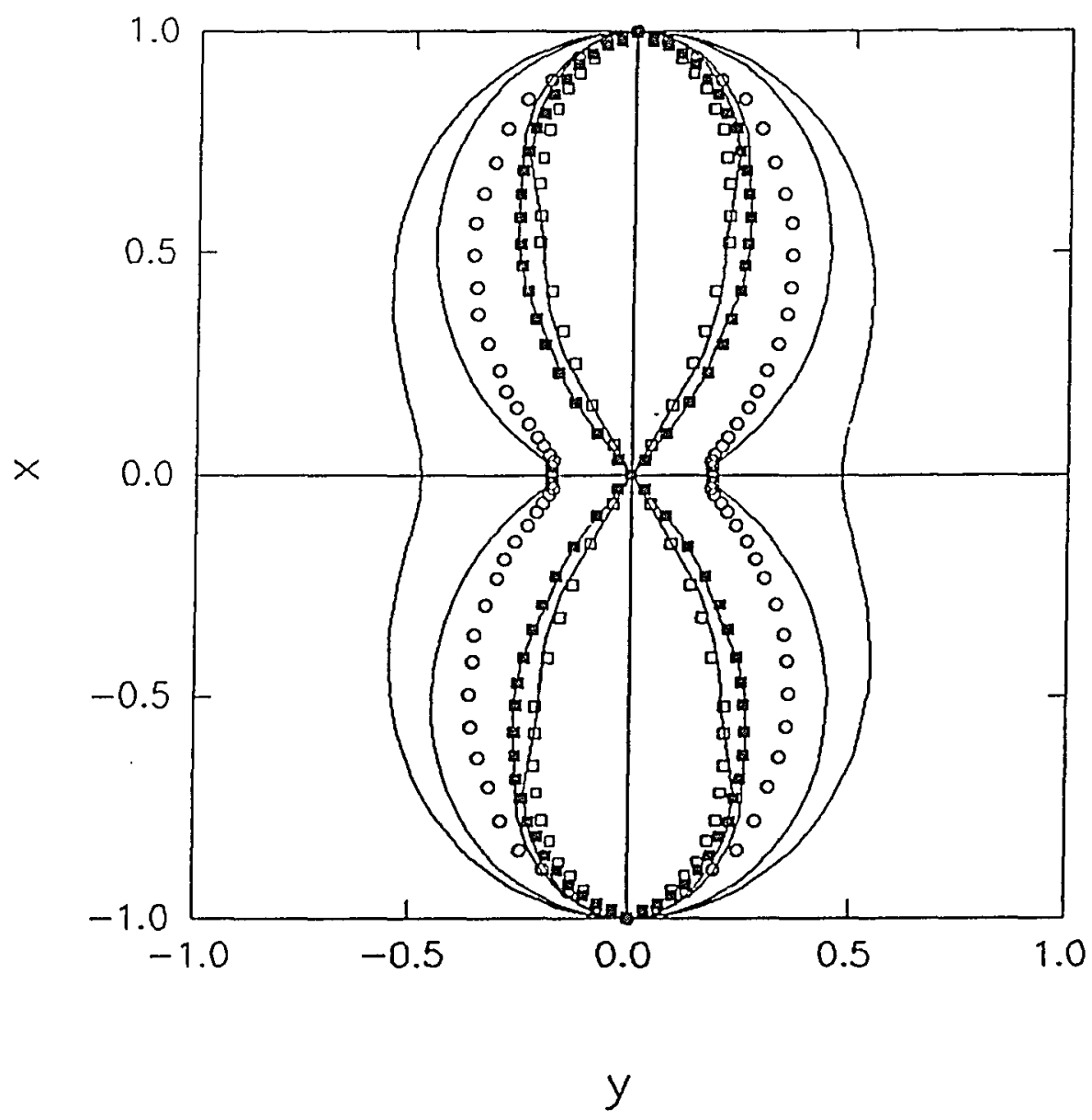

Figure 8. Normalized average power of the output pulses in polar form. The $x$ axis corresponds to the zero degree direction $(\alpha=0)$ of the pump field, which is also the direction of the signal field selected by the Brewster angle surface of the dye stream. From the inner to the outer curve the solid lines are the theoretical calculations for the threshold parameter values $r=$ $1.3,1.46,4.0$, and 10.0, respectively. The open, filled squares, and open circles are the experimental data points for $\mathrm{r}=1.3,1.46$ and 2.83, respectively. 


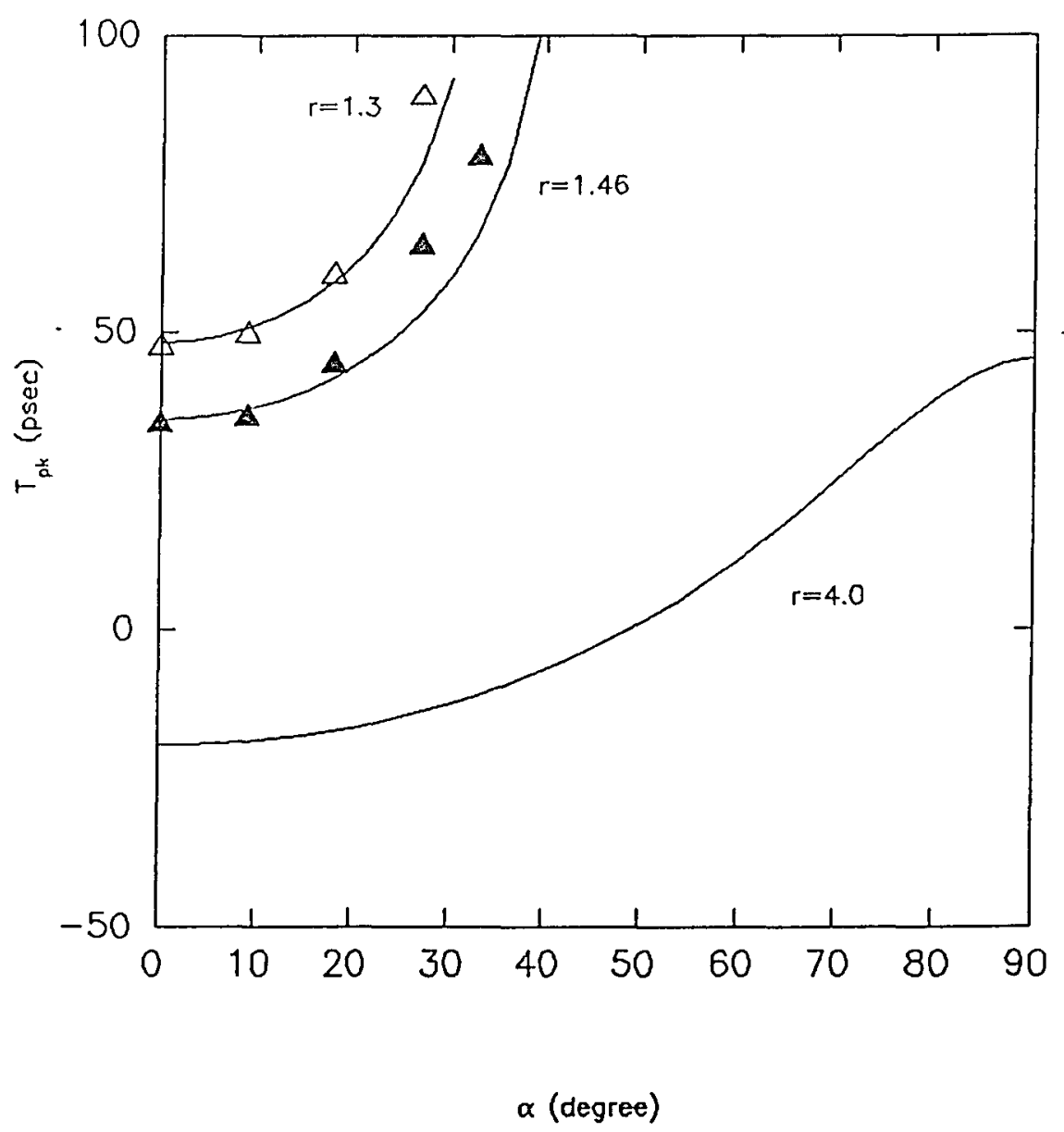

Figure 9 Peak-to-peak time delay between the pump pulse and the signal pulse versus the pump polarization angle for threshold parameter values of $r=1.3,1.46$, and 4.0 . The open and filled triangles are the experimental points for $r=1.3$ and 1.46 . 


\section{CHAPTER IV}

\section{TIMING AND DETUNING STUDIES OF A SYNCHRONOUSLY MODE-LOCKED DYE LASER}

\section{INTRODUCTION}

A convenient source of wavelength tunable ultrashort optical pulses, the modelocked dye laser has been widely studied and used for more than two decades. One popular source today is the synchronously pumped mode-locked (SPML) dye laser. State-of-the-art SPML dye lasers offer highly tunable subpicosecond pulses of reasonable power at high repetition rates $[3,44,48]$. As mentioned in previous chapters, the basic principle of synchronous pumping is that at each cycle the pump laser pulse arrives at the gain medium at the same moment as the dye laser pulse. Expressed as a cavity length condition, this requires that the length of the dye lasers be nearly an integer multiple of the pump laser length. This theoretical condition is difficult to maintain in practice due to thermal and vibration induced cavity length drift. Thus, the cavity length matching condition will not be always be satisfied precisely, and the behavior of the SPML dye laser under the circumstance of cavity length mismatch or detuning from its optimum condition becomes worthwhile to explore and understand from a technical standpoint. From a scientific standpoint, the cavity length detuning parameter is an easily adjustable experimental parameter that can be varied in the laboratory to obtain a wealth of data with which to test a theory.

To date, the effects of a finite cavity length mismatch have been explored with respect to pulse shape, pulse width, pulse buildup, peak power, average output power and 
pulse train stability, and it has been widely noted that the output pulses lengthen dramatically, lose peak power, and develop satellite pulses at lengths away from optimum [9-14, 16, 49-51]. The gain bandwidth limiting effects of an intracavity wavelength tuning filter have also been studied with a coherent model [20]. Another parameter of interest is the time of occurrence of the dye laser pulse measured relative to the pump laser pulse. This quantity has significance as an experimental test of SPML laser theory, and as a possible error signal for an active stabilization scheme. While there previously have been brief mentions of pulse timing as a function of cavity length detuning, the present work is devoted solely to the subject. Most of the previous theoretical studies of the dye laser pulse delay included one or more of the severe simplifying assumptions and approximations inherent in the use of a self consistent Gaussian pulse, rate equation models and circuit analogies $[10-12,14,51]$. All previous studies have neglected the vibrational relaxation and coherence effects which inevitably become important for very short pulsations. Only in one case has a quantitative comparison between theory and experiment been attempted [14]. Simplifications are, of course, necessary for any study of so complex a system, but models are now available which avoid most of the approximations just noted.

In this chapter, we study in detail the effect of cavity length mismatch on the occurrence time of the dye laser pulses. Experimentally, we directly measured the delay time of the peak of the dye laser pulse relative to the peak of the pump pulse with greater precision than has been achieved previously. These measurements are used to test, for the first time with no free parameters, our semiclassical theory for synchronously pumped mode-locked dye lasers, and very close agreement has been achieved. Semiclassical dye laser models have not previously been applied to the study of pulsation timing. The inclusion of semiclassical effects is important for quantitative agreement near the optimum 
cavity length detuning, which is, pragmatically, the region of most interest. In addition to this quantitative study, we also presented the results obtained from a corresponding rate equation model which could qualitatively describe the timing characteristics of the SPML laser.

\section{EXPERIMENT}

The experiments were performed using a commercially available Spectra-Physics argon ion / rhodamine $6 \mathrm{G}$ dye laser system with a cavity length of $186 \mathrm{~cm}$. The argon laser (Spectra-Physics 2020) was actively mode-locked by a fused silica acousto-optic modulator driven at about $40.25 \mathrm{MHz}$. It was operated in $\mathrm{TEM}_{00}$ transverse mode and was set up to produce a stable train of $100 \mathrm{ps}, 514 \mathrm{~nm}$ pulses with a pulse repetition frequency of $80.25 \mathrm{MHz}$. Once optimized, this argon laser system was not adjusted during the measurements, and hence provided a consistent relative clock for the timing and detuning studies.

The argon laser pumped a Spectra-Physics 375B dye laser as shown in Figure 10.. The output coupler of the dye laser was mounted on a precision translatable stage, which provided the cavity length adjustment. A Mitutoyo dial indicator permitted displacement measurements readable to $0.5 \mu \mathrm{m}$ resolution, and the overall system length error was within $\pm 1 \mu \mathrm{m}$. For our purpose, the optimum cavity length was defined as that length giving the shortest mode-locked dye laser pulses, as measured by a Spectra-Physics 409 autocorrelator. This length is nearly, but not precisely, equal to exact cavity matching between the pump laser and the dye laser [16].

A shift in the dye laser cavity length affects neither the acousto-optic modulator nor the timing position of the pump pulse. Thus the timing position of the pump pulse is a well defined reference for our measurements. The delay time of the dye laser pulse as a 


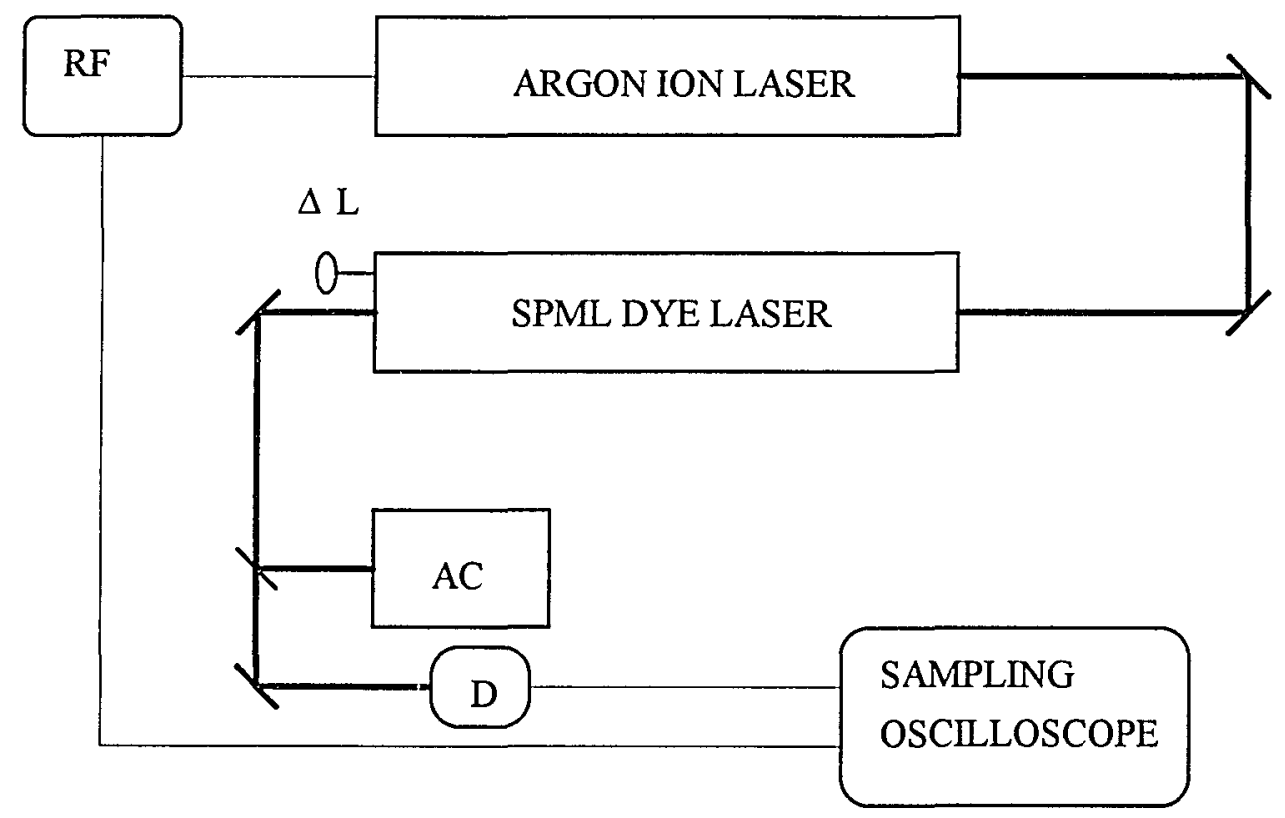

Figure 10. Schematic of the experimental set-up for study of the timing characteristics of the SPML dye laser (AC, autocorrelator; $D$, detector; $R F$, RF driver). 
function of dye laser cavity length detuning can be measured using a Tektronix 7S11 sampling oscilloscope triggered by the pump laser mode-locking signal. It made no discernible difference in our measurements whether the trigger signal came directly from the if modulator or from a photodiode monitoring the pump train. As the dye laser cavity length is changed, the dye laser pulse moves across the screen of the sampling scope, and recording this shift is our basic measurement. A typical set of raw data is shown in Figure 11. Pulse resolution of these picosecond pulses on the sampling scope is limited by the rise time of the scope ( $25 \mathrm{ps)}$ and the detector ( $35 \mathrm{ps}$ ), and by the intrinsic jitter of the system. These factors permit a determination of the timing of the peak of the waveform with an uncertainty of about \pm 10 ps as measured from the trace-width of the best sampling scope traces. The total range of detuning covered was approximately $70 \mu \mathrm{m}$. Our data of $T_{\mathrm{pk}}$ as a function of $\Delta L$ is shown in Figure 12 for two different pump powers corresponding to the threshold parameters $r=1.3$ and $r=1.7$. Experimentally, $r$ is the ratio of the actual average pump power divided by the minimum average power necessary to achieve lasing. The lower threshold parameter was reached by linearly attenuating the pump laser beam. Figure 12 reveals three basic regions of interest in $T_{p k}$ versus $\Delta L$. For negative detunings, $T_{p k}$ is insensitive to changes in $\Delta L$. For large positive $\Delta L$ 's, the dependence is almost linear. Finally, there is a steep transition region just around $\Delta L_{\mathrm{opt}}$.

\section{NUMERICAL CALCULATION}

The theoretical models we used for the numerical calculations were the semiclassical model and a corresponding rate equation model for a SPML dye laser which were derived fully in Chapter II. The semiclassical model is a set of nonlinear integrodifferential equations, Eqs. (49)-(51) and (53) with the polarization alignment angle $\alpha$ set to zero. 

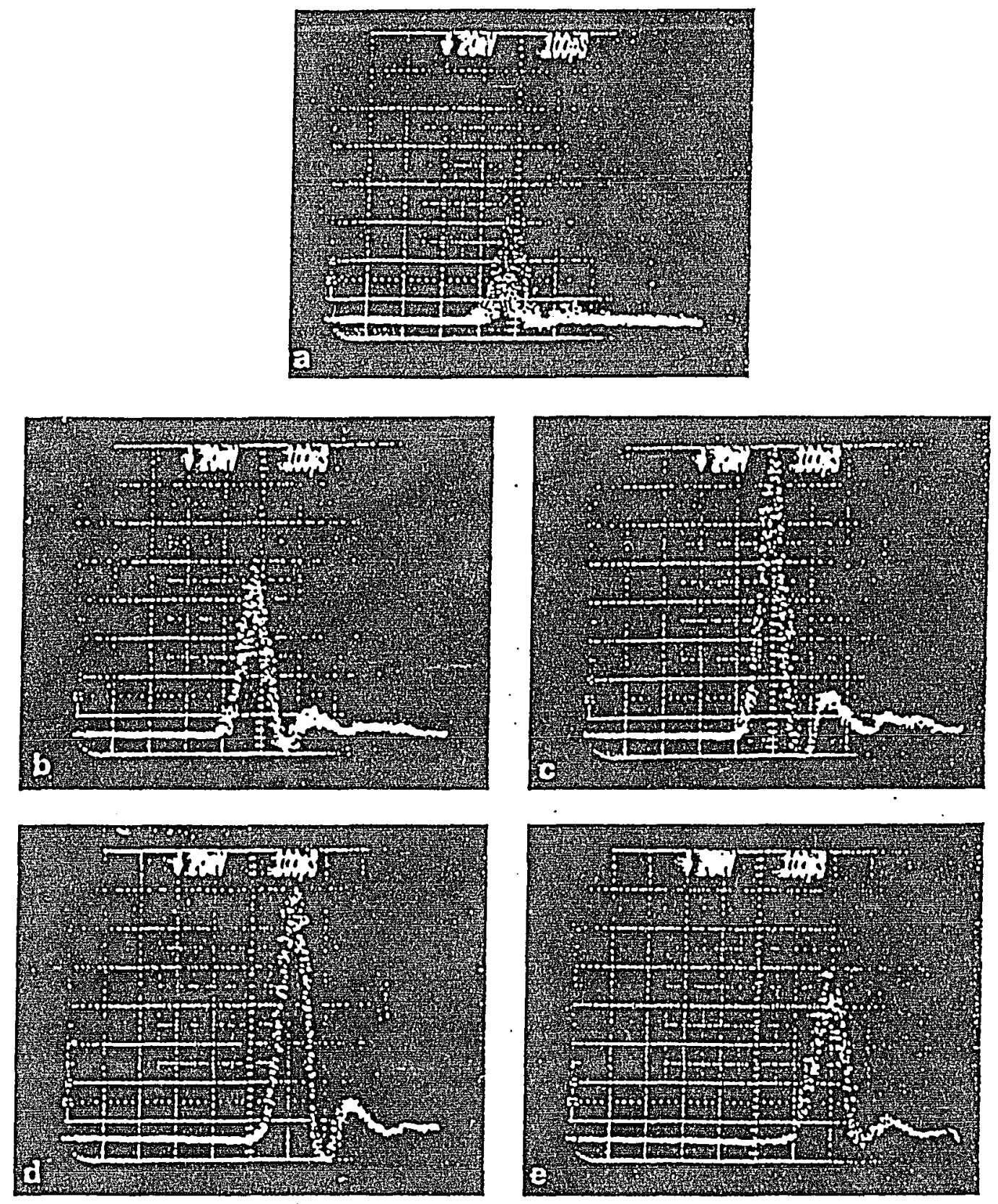

Figure 11. Sampling scope temporal traces (100 ps/div) of the modelocked dye lasers for $r=1.7$ at various cavity length detunings. Traces (a)(e) show the delay in the waveforms as the detuning is increased. The trace is unstable for cavity lengths shorter than optimum. (a) $\Delta L$ shorter than optimum by $30 \mu \mathrm{m}$; (b) $\Delta L$ shorter than optimum by $10 \mu \mathrm{m}$; (c) $\Delta L$ optimum; (d) $\Delta L$ longer than optimum by $20 \mu \mathrm{m}$; (e) $\Delta L$ longer than optimum by $40 \mu \mathrm{m}$. 


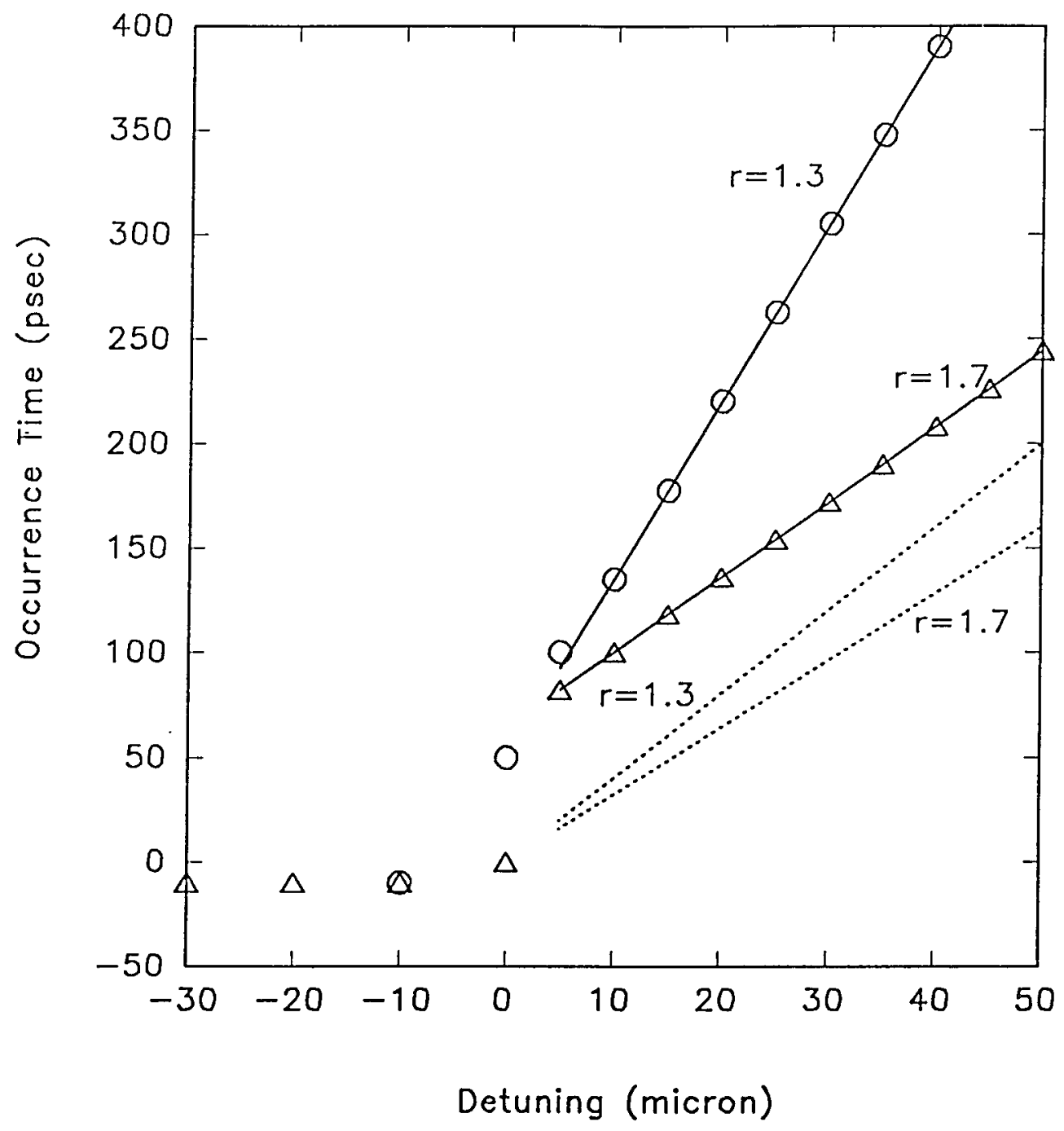

Figure 12. Experimental, numerical dependence of the SPML dye laser pulse occurrence time on the cavity length detuning. The circles are data points for $r=1.3$, the triangles are data points for $r=1.7$. The solid line are the theoretical dependence of $T_{\mathrm{pk}}$ as calculated numerically from Eqs. (69(72). The dotted lines are calculated from Eqs. (73)-(75). 


$$
\begin{aligned}
& \frac{d D}{d \tau}=-\frac{1}{\tau_{2}}\left[\left(1+\frac{\tau_{2}}{2 \tau_{1}}\right) D+\left(1-\frac{\tau_{2}}{2 \tau_{1}}\right) M+2 Q A \chi-P(\tau) \chi^{2}\right] \\
& \frac{d M}{d \tau}=-\frac{1}{\tau_{2}}\left[\left(-\frac{\tau_{2}}{2 \tau_{1}}\right) D+\left(\frac{\tau_{2}}{2 \tau_{1}}\right) M-P(\tau) \chi^{2}\right] \\
& \frac{d Q}{d \tau}=-\frac{1}{T_{s}}[Q-A D \chi] \\
& \frac{d A}{d \tau}=-\frac{L}{2 t_{c} \Delta L}\left[A-\int_{0}^{1} Q \chi d \chi\right] .
\end{aligned}
$$

The corresponding unidirectional molecular distribution, rate equation model is Eqs. (57), (58) and (60) with $Q=A D$ obtained from Eq.(59) by setting the coherence time $T_{\mathrm{s}}$ to zero.

$$
\begin{aligned}
& \frac{d D}{d \tau}=-\frac{1}{\tau_{2}}\left\{\left(1+\frac{\tau_{2}}{2 \tau_{1}}\right) D+\left(1-\frac{\tau_{2}}{2 \tau_{1}}\right) M+2 I D-P\right\} \\
& \frac{d M}{d \tau}=-\frac{1}{\tau_{2}}\left\{-\frac{\tau_{2}}{2 \tau_{1}} D+\frac{\tau_{2}}{2 \tau_{1}} M-P\right\} \\
& \frac{d I}{d \tau}=-\frac{L I}{t_{c} \Delta L}(1-D) .
\end{aligned}
$$

Quantities in the above equations have the same meaning as introduced in Chapter II. $A$ represents the normalized electric field, $D$ represents the normalized population difference, $M$ represents the normalized population sum, and $Q$ represents the normalized polarization. $I$ represents the pulse intensity which was defined as $I \equiv A^{2}$. These models explicitly include the orientation of the molecular dipoles, $\chi=\cos \theta$. This point is important for quantitative agreement between our model and experiment [16]. In general, then, Eqqs. (69)-(12) refer specially to only one orientation class and the individual classes 
contribute to the field through the integral over $\chi$ in Eq. (72). Moreover, the consideration of a finite coherence time $T_{\mathrm{s}}$ in the general semiclassical model, Eqs. (69)(72) is also important for quantitative prediction of the pulse formation especially when pulses are short. In modeling our Rhodamine $6 \mathrm{G}$ dye laser, we have used the best available values for these parameters, with $\tau_{1}=1 \mathrm{ps}, \tau_{2}=5 \mathrm{~ns}$ and $T_{\mathrm{s}}=50 \mathrm{fs}$.

The dynamical rate constant associated with the field is composed of the cavity length $L$, the cavity lifetime $t_{\mathrm{c}}$, and the cavity length mismatch $\Delta L$, which is the amount by which the dye laser cavity length exceeds that of the pump laser. In our case, $L=1.86 \mathrm{~m}$, $t_{c}=80 \mathrm{~ns}$ as calculated by considering the transmission of the output coupler, mirror diffraction and other scattering losses. The cavity length detuning $\Delta L$, is the key parameter which we vary to mimic our experiment.

The argon ion pump laser enters into this set of equations through the forcing term $\mathrm{P}(\tau)$. The pump pulse was modeled as a Gaussian profile with a full width at half maximum $\Delta \tau$ equal to $100 \mathrm{ps}$.

$$
P(\tau)=P_{0}\left(\frac{2}{\Delta \tau}\right)\left(\frac{\ln 2}{\pi}\right)^{1 / 2} \exp \left[-\left(\frac{2 \tau}{\Delta \tau}\right)^{2} \ln 2\right]
$$

The pump amplitude, $P_{0}$, can be related to the threshold parameter $r$ by determining the minimum $P_{0}$ necessary to bring the gain up to the loss level by the end of the pulse. From Eq. (72), this threshold parameter would be given by value of the ratio

$$
r=\frac{\int_{0}^{1} Q x d x}{A}
$$

where, from Eqs. (69)-(71), the value of $Q$ can be approximated by

$$
\begin{aligned}
& Q=A D x=A P_{0} x^{3} / \tau_{2} . \\
& P_{0}=5 \tau_{2} r .
\end{aligned}
$$

Similarly, for the unidirectional distribution rate equation model, it is easy to get 
$P_{0}=\tau_{2} r$.

Thus, $P_{0}$ may be obtained from the experimentally measured $r$ by using Eqs. (79) or (80).

We see that one consequence of dipole orientation effects is an inefficiency that can be quite high. In the isotropic case considered here, one must pump five times as hard to achieve a given threshold parameter as in the unidirectional distribution case.

For our calculation, we set $P_{0}$ to correspond to experimental conditions and calculated, using a Runge Kutta computer program, the time, $T_{\mathrm{pk}}$, at which the dye laser pulse is maximum. Varying $\Delta L$ allows us to predict the slope in Figure 12. The numerical results are shown in Figure 12, together with the experimental data. The vertical scale is taken from the calculated $T_{\mathrm{pk}}$ and hence is absolute, not relative. For negative detunings, the SPML dye laser pulse returns to the gain medium before the pump overcomes the loss. Hence the SPML dye laser pulse is delayed each cycle until the lasing threshold condition is met. Physically, this is why $T_{\mathrm{pk}}$ is insensitive to variations in $\Delta L$ for cavity lengths shorter than optimum. Our model and our experiments show that the pulse is unstable for negative $\Delta L s$. An important implication of both the experimental data and the theoretical results is that for positive length detunings, the pulse delay $T_{\mathrm{pk}}$ is an almost linear function of detuning. Also by comparing the curves of the semiclassical model and the unidirectional rate equation model, one can find that the unidirectional rate equation model predicts a flatter slope of $T_{\mathrm{pk}}$ versus $\Delta L$. For the consideration of pulse stability, it seems that the unidirectional rate equation model may over-estimate the stability of the SPML dye laser system.

\section{CONCLUSION}

We have explored the timing relationship between a synchronously pumped modelocked dye laser pulse and its pump pulse through experiments and theory. We have 
shown that our semiclassical theory for a SPML dye laser predicts a nearly linear timing shift of the dye laser pulse with cavity length detuning, and this result is in close quantitative agreement with our experimental data.

Our cbservation that a triggering signal from the RF modulator is as effective as a triggering signal from the pump pulse train implies that the major portion of the jitter on the system comes from the electronics of the RF modulator. For low absolute jitter applications, such as the electro-optic sampling of electronic circuits, it would be most advantageous to the system's performance to control the timing jitter in the RF modulator circuitry.

Our theory and experiments show that the dependence of $T_{\mathrm{pk}}$ on $\Delta L$ weakens as $r$ becomes larger. Thus we may conclude that for a fixed, optimum cavity length mismatch, the timing jitter of a SPML dye laser may be reduced by increasing the pump power. Alternatively, at low pump power levels, the occurrence time may be used as an error signal for an active feedback stabilization scheme since there is an abrupt shift in $T_{\mathrm{pk}}$ versus $\Delta L$ near the optimum detuning. 


\section{CHAPTER V}

\section{ULTRASHORT PULSE PROPAGATION IN DYE LASER AMPLIFIERS}

\section{INTRODUCTION}

Interests in ultrashort pulse amplification has grown rapidly during the past few years, and because of their high gain and broad gain spectrum, liquid-dye based laser amplifiers are usually considered to be the most attractive amplifying media. Amplification of pulses as short as $26 \mathrm{fs}$ has been reported [26]. Many different pump sources have been employed in dye laser amplifiers including cw YAG lasers [52-55], argon ion lasers [56, 57], copper vapor lasers, and $\mathrm{XeCl}$ excimer lasers $[58,59]$. Some of these systems were used to obtain pulses at high repetition rates, while others emphasized high peak powers. However, they all try to maintain pulse widths of less than 100 femtosecond. In spite of the rapid development of experimental techniques for ultrashort optical pulse amplification, the theory of this subject seems to have lagged behind. To date, only a few theoretical studies of picosecond or subpicosecond dye laser pulse amplifications have been reported [60-64]. These rate equation models have explained many aspects of dye laser amplifier performance in the picosecond regime. However, they meet serious difficulties as the optical pulses go to the subpicosecond and femtosecond regimes.

First, as these models usually originate from basic rate equation concepts, they may not consider the molecular vibrational relaxation time, which is on the order of a picosecond. This relaxation time has already been shown to be of importance in interpreting the evolution of picosecond laser pulses [16]. In the subpicosecond or 
femtosecond regime, the effects of this relaxation time are expected to be even more substantial.

Second, in these models the coherence time or the dephasing time of the dye molecules of the amplifying medium was not taken into account. Depending on solvents, spectral positions and sample deterioration, this time has been found to lie in the range of 20 fs to 2 ps, [16], which might be unimportant for picosecond pulses but not for femtosecond pulses. Rate equation models describe the light-matter interaction based on the assumption that the polarization varies instantaneously with the field, or in other words that the coherence time is zero. Such an assumption may be valid when the amplified pulses are much longer than the coherence time, i.e., the signal field varies much slower than the dephasing time. However, when the signal pulse width approaches the coherence time, rate equation models fail to explain the light-matter interaction adequately. Coherence time effects have been studied in excimer amplifying media such as $\mathrm{KrF}$ and $\mathrm{XeCl}$. [21-24]. Significant differences, especially in the amplified pulse temporal evolution, were found between the results obtained with and without consideration of the coherence time. As dye laser media have a much different gain spectrum and energy structure, those models can not be used to describe the complex nonlinear processes that take place inside dye laser amplifiers.

Third, in order to characterize the dye medium, the molecular orientational distribution must be included in the model. With parallel pump and signal polarizations and modest pumping levels, a unidirectional orientational distribution can provide qualitative insight into some aspects of dye laser amplification. However, inclusion of the

more realistic isotropic distribution has been shown to be necessary for quantitative interpretations or predictions of synchronously pumped mode-locked dye laser oscillators $[16]$. 
Using the general semiclassical model, Eqs. (23)-(26), derived in Chapter II, which was based on the density matrix equations and Maxwell's equation, we have studied in detail the dynamic behavior of ultrashort pulses propagating in a dye laser amplifier. In

addition, both the rate equation model and the unidirectional molecular distribution model were employed. Numerical results were compared to see the validity of those simplified models.

\section{SIMPLIFICATIONS OF THE THEORY}

\section{Semiclassical Model}

$$
\begin{aligned}
& \frac{\partial D}{\partial t}=-\frac{1}{\tau_{2}}\left\{\left(1+\frac{\tau_{2}}{2 \tau_{1}}\right) D+\left(1-\frac{\tau_{2}}{2 \tau_{1}}\right) M+2 Q A x-P\left[\chi^{2}\left(1-\frac{3 \sin ^{2} \alpha}{2}\right)+\frac{\sin ^{2} \alpha}{2}\right]\right\}, \\
& \frac{\partial M}{\partial t}=-\frac{1}{\tau_{2}}\left\{-\frac{\tau_{2}}{2 \tau_{1}} D+\frac{\tau_{2}}{2 \tau_{1}} M-P\left[\chi^{2}\left(1-\frac{3 \sin ^{2} \alpha}{2}\right)+\frac{\sin ^{2} \alpha}{2}\right]\right\}, \\
& \frac{\partial Q}{\partial t}=-\frac{1}{T_{s}}(Q-A D \chi), \\
& \frac{\partial A}{\partial z}+\frac{1}{v} \frac{\partial A}{\partial t}=-\frac{\gamma_{s}}{2}\left(A-\int_{0}^{1} Q \chi d \chi\right) .
\end{aligned}
$$

As mentioned in Chapter II, $D$ is a normalized population difference, $M$ is a normalized population sum, $Q$ is a normalized polarization, $A$ is a normalized signal electric field, and $P$ is a normalized pump rate. $T_{s}$ is the coherence time, $\gamma_{s}$ is the distributed loss, and $\chi=\cos \theta$ where the variable $\theta$ measures the angle of a class of signal dipoles with respect to the field polarization direction. $\alpha$ measures the misalignment between the pump field and the signal field. 
Simplifications

In order to have the highest interaction efficiency, the pump field needs to have the same polarization as the signal field, which was previously demonstrated in detail in a synchronously pumped mode-locked dye laser systems [28]. In the present case, we will only consider the parallel alignment of the pump field and signal field. Therefore, one may substitute $\alpha=0$ into Eq. (81)-(84), and it is helpful to introduce a new set of coordinate variables as

$\tau=t-\frac{z}{v}$

$\zeta=\gamma_{s} z$

With some simple mathematical simplifications, one carı express Eqs. (81)-(84) in terms of the new coordinate variables as

$$
\begin{aligned}
& \frac{\partial D}{\partial \tau}=-\frac{1}{\tau_{2}}\left\{\left(1+\frac{\tau_{2}}{2 \tau_{1}}\right) D+\left(1-\frac{\tau_{2}}{2 \tau_{1}}\right) M+2 Q A x-P x^{2}\right\}, \\
& \frac{\partial M}{\partial \tau}=-\frac{1}{\tau_{2}}\left\{-\frac{\tau_{2}}{2 \tau_{1}} D+\frac{\tau_{2}}{2 \tau_{1}} M-P x^{2}\right\}, \\
& \frac{\partial Q}{\partial \tau}=-\frac{1}{T_{s}}(Q-A D x), \\
& \frac{\partial A}{\partial \zeta}=-\frac{1}{2}\left(A-\int_{0}^{1} Q x d x\right) .
\end{aligned}
$$

It is also helpful to introduce a pump threshold parameter $r$. A threshold condition may be defined as that when a steady-state signal sees zero distributed gain along an unsaturated amplifier. Therefore, from Eqs. (87)-(89), one finds

$$
0=-\frac{1}{\tau_{2}}\left\{\left(1+\frac{\tau_{2}}{2 \tau_{1}}\right) D+\left(1-\frac{\tau_{2}}{2 \tau_{1}}\right) M+2 Q A x-P x^{2}\right\},
$$


$0=-\frac{1}{\tau_{2}}\left\{-\frac{\tau_{2}}{2 \tau_{1}} D+\frac{\tau_{2}}{2 \tau_{1}} M-P x^{2}\right\}$,

$0=-\frac{1}{T_{s}}(Q-A D x)$

$\frac{\partial A}{\partial z}=-\frac{\gamma_{s}}{2}\left(A-\int_{0}^{1} Q x d x\right)$

Eq. (93) has the solution

$Q=A D x$,

and Eqs. (91) and (92) have the solutions

$D=P x^{2}\left(1-\frac{\tau_{1}}{\tau_{2}}\right)$

$M=D+\frac{2 \tau_{1}}{\tau_{2}} P x^{2}=P x^{2}\left(1+\frac{\tau_{1}}{\tau_{2}}\right)$.

Substitution of Eq.(95) into Eq. (94) using Eq. (96) leads to

$$
\begin{aligned}
\frac{\partial A}{\partial z} & =-\frac{\gamma_{s}}{2} A+\frac{1}{2} \int_{0}^{1} A D x^{2} d x \\
& =-\frac{\gamma_{s}}{2}\left[A-A P\left(1-\frac{\tau_{1}}{\tau_{2}}\right) \int_{0}^{1} x^{4} d x\right] \\
& =-\frac{\gamma_{s}}{2} A\left[1-\frac{P}{5}\left(1-\frac{\tau_{1}}{\tau_{2}}\right)\right]
\end{aligned}
$$

In terms of the light intensity $I=A^{2}$, Eq. (98) can be expressed

$$
\frac{\partial I}{\partial z}=-\gamma_{s}\left[1-\frac{P}{5}\left(1-\frac{\tau_{1}}{\tau_{2}}\right)\right] I .
$$


As mentioned above, threshold may be defined as the condition when the distributed gain for the intensity is zero. Thus a threshold pump rate can be obtained from Eq. (99):

$$
P_{\text {threshold }}=\frac{5 \tau_{2}}{\tau_{2}-\tau_{1}} .
$$

The pump threshold parameter $r$ describes the degree of pumping above threshold. In terms of the threshold parameter, any pump rate $P$ can be expressed as $P=r P_{\text {threshold }}$.

\section{NUMERICAL RESULTS}

By numerically solving Eqs. (87)-(90), one can obtain all of the parameters concerning ultrashort pulse propagation in a dye laser amplifier. The physical situation of a pulse propagating in an amplifier is illustrated in the space-time diagram of Figure 13, which is similar to a pulse propagation description developed for maser amplifiers [65]. It is seen that the new time coordinate $\tau$ remains constant for any part of the signal as it passes from the input to the output. For instance, the leading edge of a pulse may be characterized everywhere by $\tau=\tau_{0}$, whereas in real time it would pass the input at $t=t_{0}$ and the output at $t=t_{0}+L / v$ where $L$ is the amplifier length and $v$ is the speed of light.

The figure also suggests that the physical situation at a point $(\zeta, \tau)$ is determined by all interactions which happened earlier, i.e., from time $-\infty$ to $\tau$ and closer, i.e., from 0 to $\zeta$. Such a problem can not be specified unless two boundary conditions are set up. One is the initial condition which specifies the situation for values of space $0<\zeta<L$ at initial time $\tau$ $=-\infty$. The second boundary condition is the input condition which specifies the situation for time $-\infty<\tau<\infty$ at the input $\zeta=0$. With these two boundary conditions, the equations permit a unique evaluation of the variables at any position $\zeta$ and time $\tau$. 


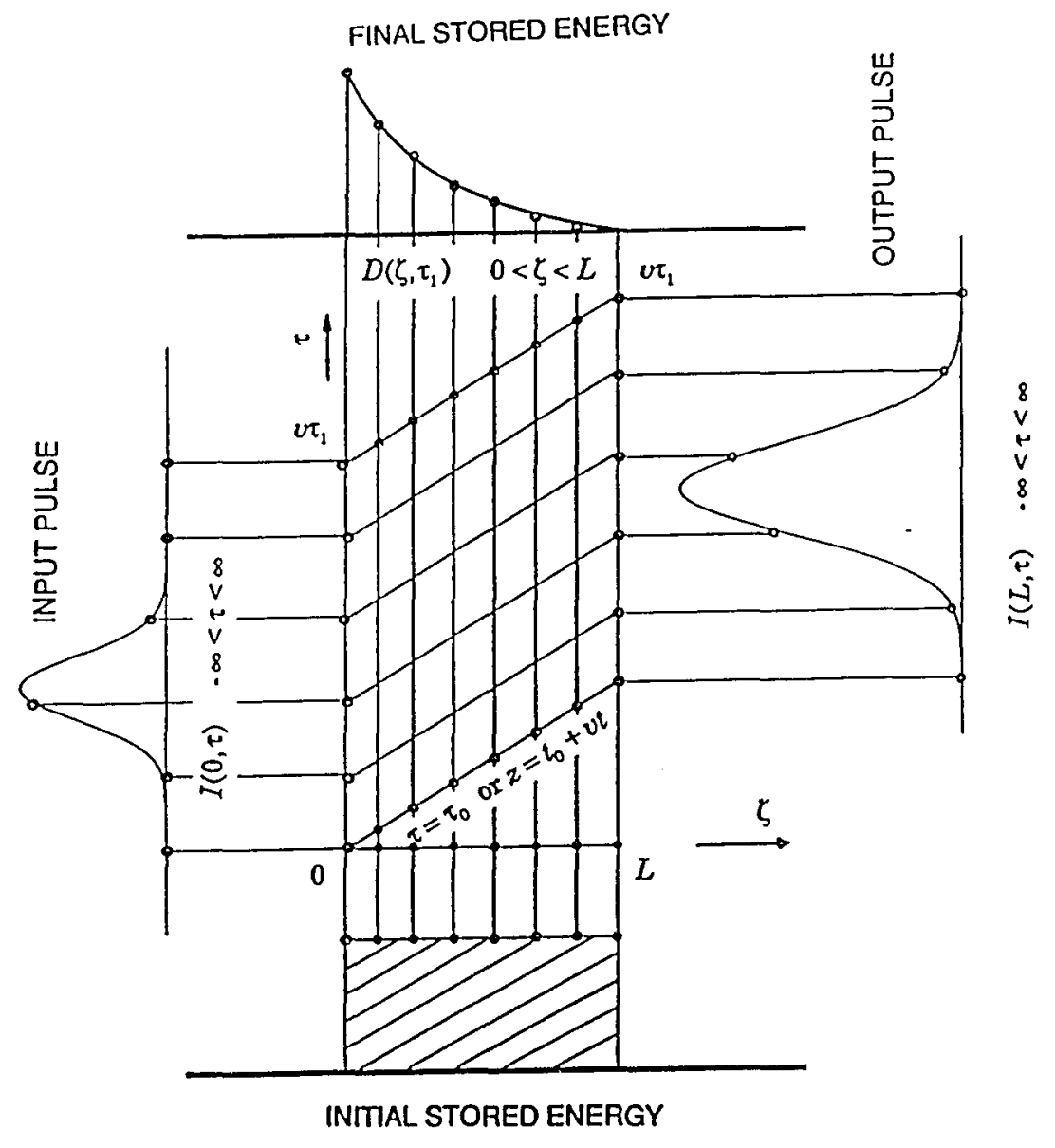

$$
D\left(\zeta, \tau_{0}\right) \quad 0<\zeta<L
$$

Figure 13. Schematic diagram of a pulse propagating in a dye laser amplifier and the gain process by depletion of stored energy; also illustrated are the evolution in time $\tau$ and space $\zeta$. 
The two boundary conditions for the present problem include the initial condition for the population difference $D$, population sum $M$, polarization $Q$, and signal field $A$ or intensity $I$. As the amplified spontaneous emission (ASE) is neglected here, $D$ and $M$ are determined by Eqs. (87)-(88), which are

$D(\zeta, \tau=-\infty)=P(\zeta) \chi^{2}\left(1-\frac{\tau_{1}}{\tau_{2}}\right) \quad$ for $0<\zeta<L$ and zero anywhere else

$M(\zeta, \tau=-\infty)=P(\zeta) \chi^{2}\left(1+\frac{\tau_{1}}{\tau_{2}}\right) \quad$ for $0<\zeta<L$ and zero anywhere else.

The initial time here is defined as the moment just before the input pulse enters the amplifier. As ASE is assumed to be negligible, the initial condition for the electric field is zero, as is the initial condition for the polarization.

The other condition describes the input signal

$A(\zeta=0, \tau)=\left\{W\left(\frac{2}{\Delta \tau_{i}}\right)\left(\frac{\ln 2}{\pi}\right)^{1 / 2} \exp \left[-\left(\frac{2 \tau}{\Delta \tau_{i}}\right) \ln 2\right]\right\}^{1 / 2}$,

where a Gaussian profile of the input pulse was assumed. $W$ is the pulse energy density. The initial condition for the other equations can not be specified directly. However, after the electric field or the intensity of the pulse is specified, $D(\zeta=0, \tau), M(\zeta=0, \tau)$, and $Q(\zeta=0, \tau)$ can be resolved from Eqs. (87)-(89), which are one-dimensional, coupled firstorder differential equations. A fourth-order Runge-Kutta method was used to get these numerical solutions.

The signal at arbitrary space and time $(\zeta, \tau)$ was computed as illustrated in Figure 13. At any constant $\zeta$, the signal at time $\tau$ was determined by the population difference, population sum, and the polarization at that time and earlier. At any time, the signal at a point $\zeta$ was determined by the interaction before that position. A recursive formula for 
space and time was used for the entire time range and for the position along the amplifier cell.

Although an arbitrary pumping rate can be used in the model, a constant pumping rate has been assumed for the present problem. This is valid as long as the pumping is approximately uniform along the amplifier cell, and the pump rate varies slowly compared to the signal. These conditions are satisfied for most practical amplifier setups. The parameters related to Rhodamine $6 \mathrm{G}$ include the vibrational relaxation time $\tau_{1}=1 \mathrm{ps}$, the fluorescence decay time $\tau_{2}=5 \mathrm{~ns}$, and the coherence time $T_{\mathrm{s}}=50 \mathrm{fs}$.

\section{$\underline{\text { General Results }}$}

A normalized energy density of $\mathrm{W}=1 \times 10^{-8} \mathrm{sec}$ was employed for the input pulse, which represents an input pulse energy of about $10 \mathrm{~nJ}$ if the beam has a diameter of $2 \mathrm{~mm}$. The FWHM of the input pulse is chosen as $1 \mathrm{ps}, 100 \mathrm{fs}$ and $10 \mathrm{fs}$, the pump threshold parameter $r$ is $1 \times 10^{4}$, and the amplifier cell length is $1 \mathrm{~cm}$. With these parameters, solutions of Eqs. (87)-(90) have been carried out.

\section{$\underline{\text { Rate Equation Approximation }}$}

In the rate equation limit where the polarization varies simultaneously with the electric field, the coherence time $T_{\mathrm{s}}$ can be treated as zero. As a result, Eq. (89) has the solution

$Q=A D \chi$.

If this solution is substituted into Eqs. (87)-(88) and (90), one obtains

$$
\begin{aligned}
& \frac{\partial D}{\partial \tau}=-\frac{1}{\tau_{2}}\left\{\left(1+\frac{\tau_{2}}{2 \tau_{1}}\right) D+\left(1-\frac{\tau_{2}}{2 \tau_{1}}\right) M+2 A^{2} \chi^{2}-P \chi^{2}\right\}, \\
& \frac{\partial M}{\partial \tau}=-\frac{1}{\tau_{2}}\left\{-\frac{\tau_{2}}{2 \tau_{1}} D+\frac{\tau_{2}}{2 \tau_{1}} M-P \chi^{2}\right\},
\end{aligned}
$$


$\frac{\partial A}{\partial \zeta}=-\frac{A}{2}\left(1-\int_{0}^{1} D \chi^{2} d \chi\right)$

In this limit, a set of three nonlinear coupled differential equations, instead of four as in the above sections, is employed to describe the light-matter interaction in a dye laser amplifier. Figure 14 illustrates the pulse intensity (normalized to its peak value) as a function of time for both the general semiclassical model and the rate equation approximation at small signal gain lengths $g l$ of 10,20,30,40, and 50. Parts (a), (b), and (c) correspond to input pulse widths of $1 \mathrm{ps}, 100 \mathrm{fs}$ and $10 \mathrm{fs}$, respectively. The solid lines represent the general semiclassical model and the dotted lines represent the rate equation model. Different time scales should be noticed in these figures. The time corresponding to the peak of the input pulses for all of the cases is at zero. As the input pulse propagates in the amplifier, the leading edge of the pulse experiences larger gain than the tail due to the gain saturation, and thus the peak of the pulse travels faster than the speed of light. The sharpening of the leading edge and the pulse broadening can be seen clearly, which are some of the well-known characteristics of pulse propagation inside an amplifier predicted by rate equation models as seen in case (a). It is seen that the semiclassical model and the rate equation model predict similar intensity temporal profiles in this range where the signal pulse width is long compared to the coherence time. When the pulse width becomes shorter as in case (b), however, the difference appears more obvious and the rate equation model begins to show some inaccuracies. The pulse width predicted by the semiclassical model is much shorter than that predicted by rate equations. The pulse delay time is also different but the peak is still moving forward. When the input pulse becomes much shorter, as in case (c), the peak may even move slower than the speed of light, and the pulse width may become smaller as the pulse propagates along the amplifier. 

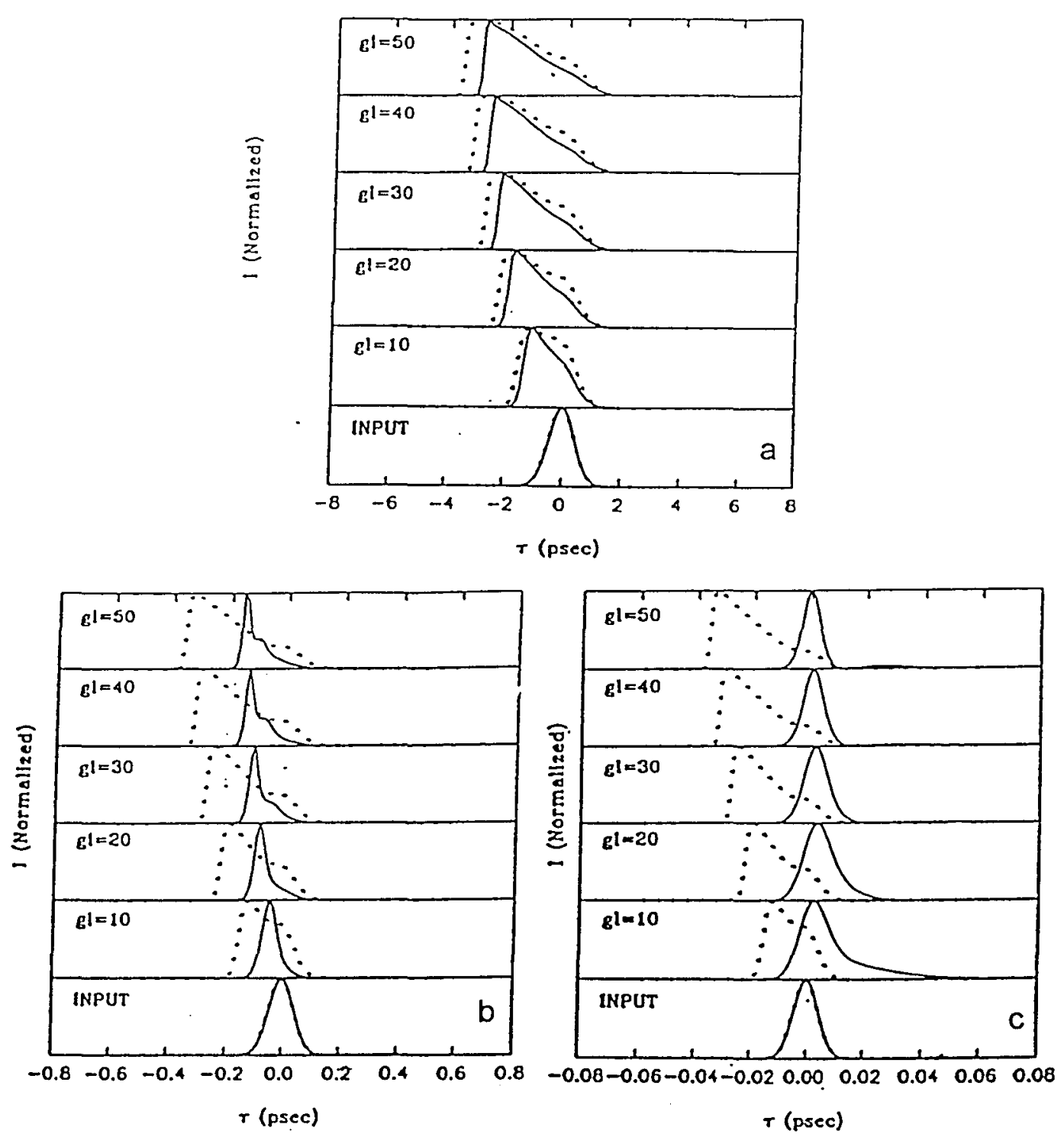

Figure 14. Normalized intensity temporal profiles of amplified pulses with initial small signal gain-length product from 10 to 50 based on the general semiclassical model (solid lines) given in Eqs. (87)-(90) and rate equation model (dotted lines) given in Eqs. (106)-(108). A normalized input pulse energy density of $1 \times 10^{-3} \mathrm{sec}$ and a pump threshold parameter of $10^{4}$ were employed. The input pulse widths are (a) $1 \times 10^{-12} \mathrm{sec}$, (b) $1 \times 10^{-13} \mathrm{sec}$, and (c) $1 \times 10^{-14} \mathrm{sec}$. Different time scales are employed in cases (a), (b), and (c). 
It can be seen from the right hand side of the field wave equation of Eq. (90) that the field can not be separated out for the general semiclassical model, and the conventional idea of intensity gain has little meaning. The population difference between the laser transition levels is of more significance. The curves in Figure 15 show that the temporal behavior of the integrated (over the entire solid angle) population difference, and the time scales for parts (a), (b), and (c) are the same as those in Figure 14. The Isolid lines represent the semiclassical model, and the dotted lines represent the rate equation model. The population difference is always positive in the rate equation model but can be negative in some time regimes in the semiclassical model due to the coherence time effects. When the pulse width becomes comparable to the coherence time, the induced polarization can not respond instantaneously to changes in the population inversion and electric field but is delayed due to the finite coherence time. As a result, the pulse can even be amplified when the population difference is negative. When the polarization changes from positive to negative or changes from a larger to a smaller value, the amplifier may turn into an absorber. In this case, the amplifying medium behaves like a saturable absorber which quenches the tail part of the pulse. Thus the pulse peak slows down and the pulse width can be shortened as the pulse is propagating in the amplifier.

The integrated energy gain versus the small signal gain-length product $g l$ is plotted in Figure 16 for the general semiclassical model (a) and for the rate equation model (b). Even though the temporal behavior of the amplified pulses may vary dramatically, the energy gain curves predicted by both models for pulses with different pulse widths are almost the same. The same energy and shorter pulses predicted by the semiclassical model imply higher peak intensities in the semiclassical model. For the input pulse we have used, the energy gain saturation starts at around $g l=10$ for all cases. 

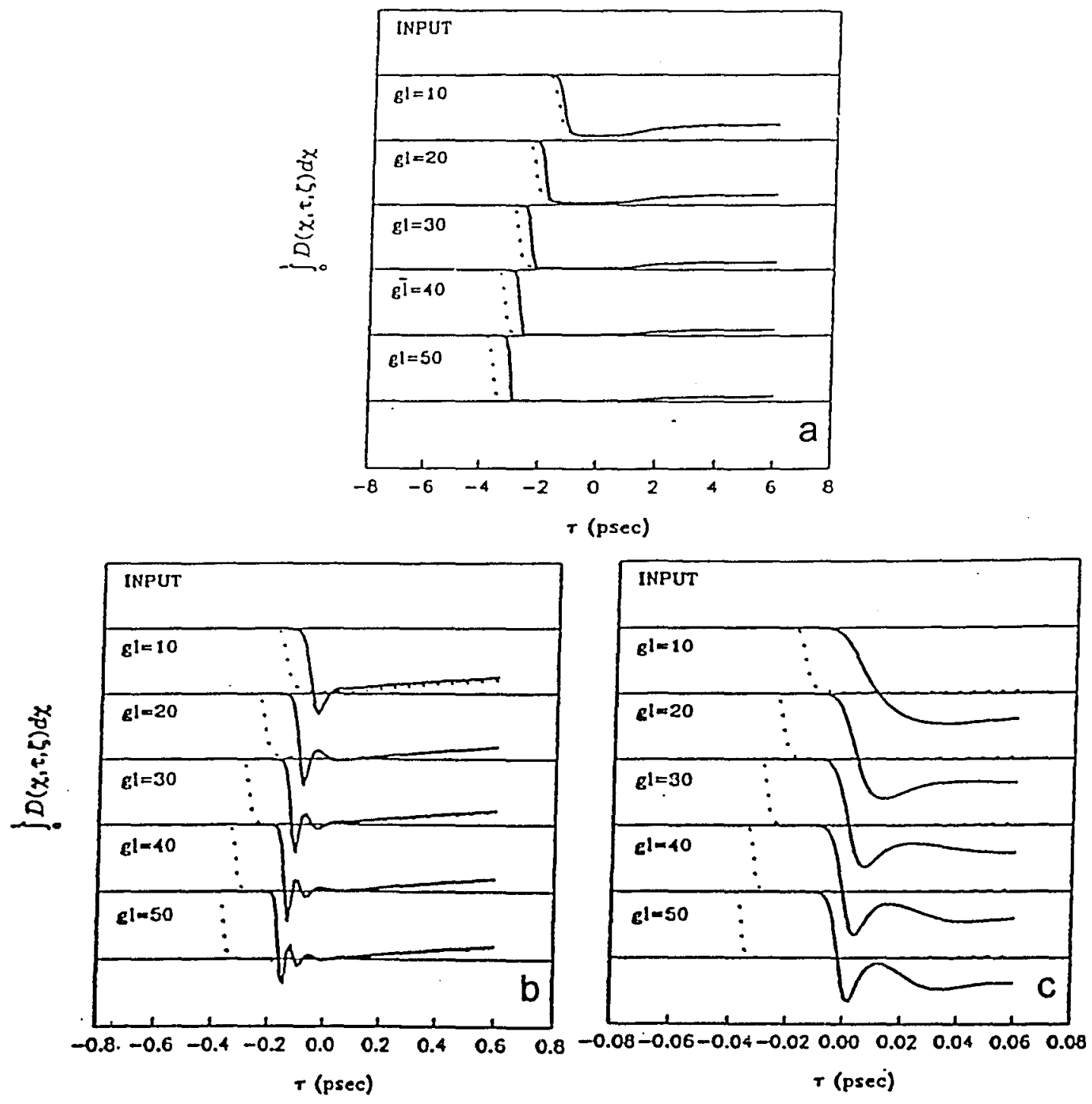

Figure 15. Normalized integrated (over the entire solid angle) population differences as a function of time. Solid lines are the general semiclassical model, and dotted lines are the rate equation model. The input pulse widths are (a) $1 \times 10^{-12} \mathrm{sec},(\mathrm{b}) 1 \times 10^{-13} \mathrm{sec}$, and (c) $1 \times 10^{-14} \mathrm{sec}$. 

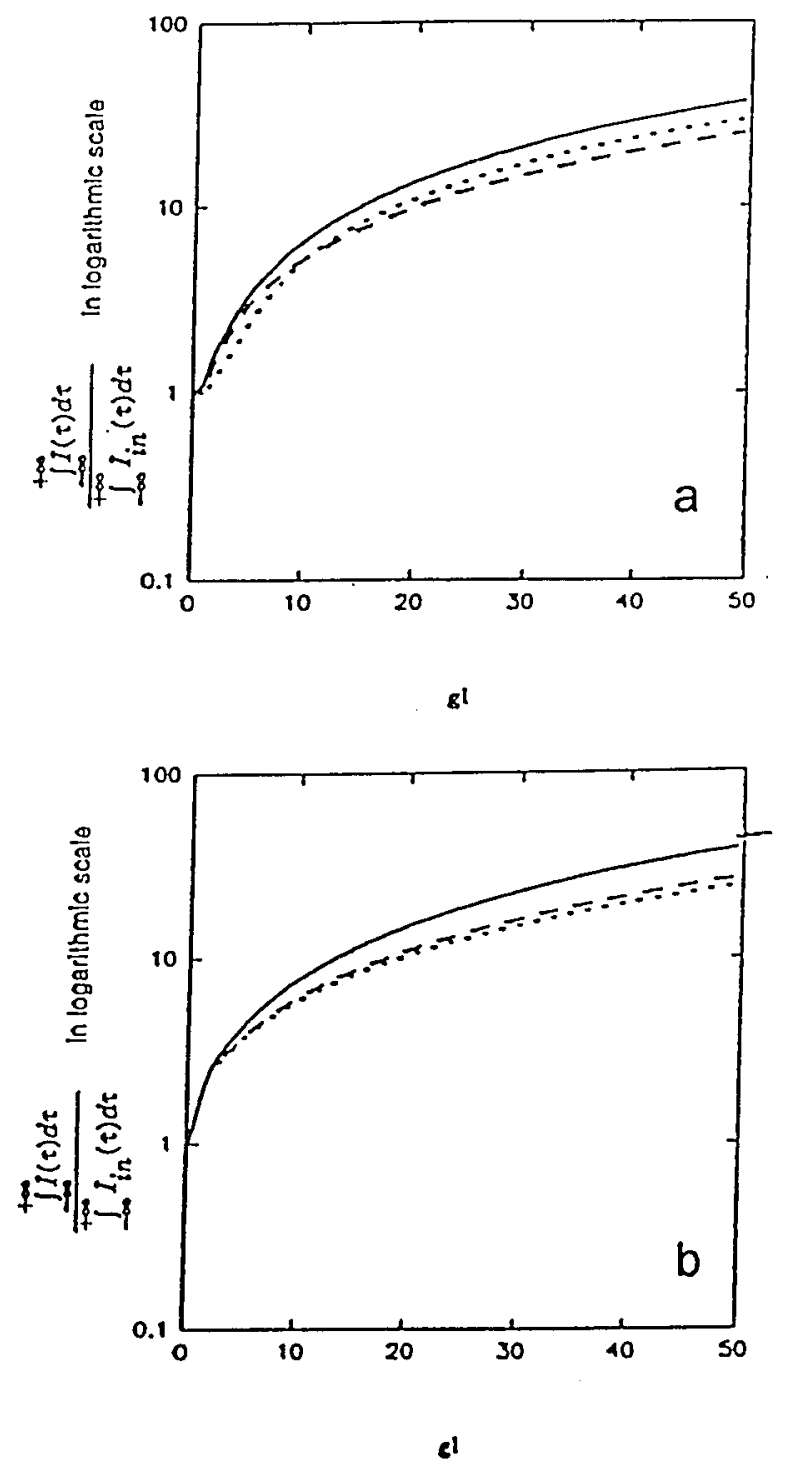

Figure 16. Integrated pulse energy gain based on the general semiclassical model (a) and rate equation model (b) as a function of small signal gainlength product for input pulse widths of $1 \times 10^{-12} \mathrm{sec}$ (solid lines), $1 \times 10^{-13} \mathrm{sec}$ (dashed lines), and $1 \times 10^{-14} \mathrm{sec}$ (dotted lines). Other parameters are the same as in Figure 14. 


\section{$\underline{\text { Unidirectional Molecular Distribution }}$}

For modest pump power, the unidirectional molecular distribution is a common way to simplify the interaction in dye lasers as well as to use less computer time. It was concluded in Ref. [16] that the unidirectional distribution approximation could qualitatively describe the synchronously pumped mode-locked dye laser oscillator.

By simplifying Eqs. (87)-(90), one can get a set of equations for the unidirectional case:

$$
\begin{aligned}
& \frac{\partial D}{\partial \tau}=-\frac{1}{\tau_{2}}\left\{\left(1+\frac{\tau_{2}}{2 \tau_{1}}\right) D+\left(1-\frac{\tau_{2}}{2 \tau_{1}}\right) M+2 Q A-P\right\}, \\
& \frac{\partial M}{\partial \tau}=-\frac{1}{\tau_{2}}\left\{-\frac{\tau_{2}}{2 \tau_{1}} D+\frac{\tau_{2}}{2 \tau_{1}} M-P\right\}, \\
& \frac{\partial Q}{\partial \tau}=-\frac{1}{T_{s}}(Q-A D), \\
& \frac{\partial A}{\partial \zeta}=-\frac{1}{2}(A-Q) .
\end{aligned}
$$

The parameters used here were the same as those above. However, the threshold pump rate required to give zero distributed gain for the signal intensity is changed to

$$
P_{\text {threshold }}=\frac{\tau_{2}}{\tau_{2}-\tau_{1}}
$$

The pump threshold in the unidirectional case is a fifth of that for the isotropic distribution. This is reasonable as in the unidirectional case all the molecular dipoles are aligned in the direction of the pump and signal fields, and all the dipoles contribute to the interaction. The pump rate can be expressed in terms of $r$ as

$P=r P_{\text {threshold }}$. 
The results of a unidirectional molecular distribution model for the pulse propagation in a dye laser amplifier are compared with those of the general semiclassical model, which employed the isotropic molecular distribution, in the following figures. Figure 17 shows the intensity profiles as a function of time for input pulse widths of $1 \mathrm{ps,}$ $100 \mathrm{fs}$, and $10 \mathrm{fs}$. It is found that the pulses in the unidirectional distribution case are similar to those in the general semiclassical model. The echo pulses in the unidirectional case are farther apart and hold more energy. Intuitively, the unidirectional distribution should lead to larger intensities as all the dipoles are aligned with the signal and pump fields. However, it should be noticed that to reach the same level above threshold, the pump for an isotropic distribution has be five times as large as for a unidirectional distribution. Thus, the isotropic distribution can give higher intensity than the unidirectional distribution if they are at the same pump level above threshold.

Figure 18 shows the population difference for the isotropic distribution and the unidirectional distribution. It is found that coherence time effects in the unidirectional case are stronger. The pulse energy gains are still similar for both cases as illustrated in Figure 19.

\section{CONCLUSION}

A theoretical model has been developed for the propagation of ultrashort pulses in dye laser amplifiers. In comparison with the results from rate equation models, one finds that a semiclassical model including the finite coherence time, isotopic molecular distribution, and fast vibrational relaxation time should be much more accurate in characterizing nonlinear processes in the amplifier. Although some pulse amplification systems employ a saturable absorber together with the amplifier to compress the pulses, it is clear from this investigation that such approaches should be preceded by more detailed 

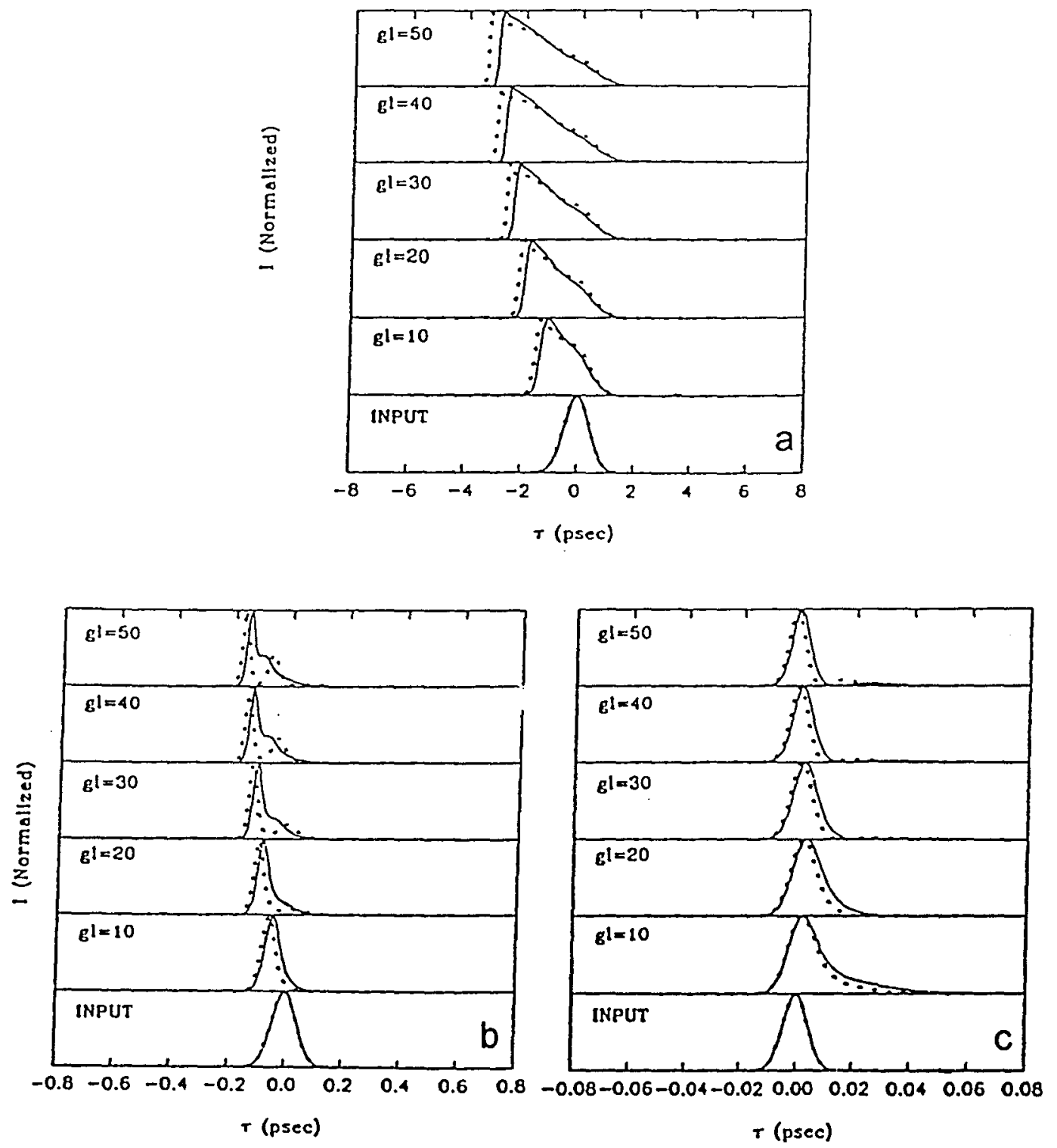

Figure 17. Normalized intensity temporal profiles of amplified pulses with initial small signal gain-length product from 10 to 50 based on the general (isotropic molecular distribution) semiclassical model (solid lines) given in Eqs. (87)-(90) and the unidirectional molecular distribution model (dotted lines) given in Eqs. (109)-(112). Parameters are the same as in Figure 14. 

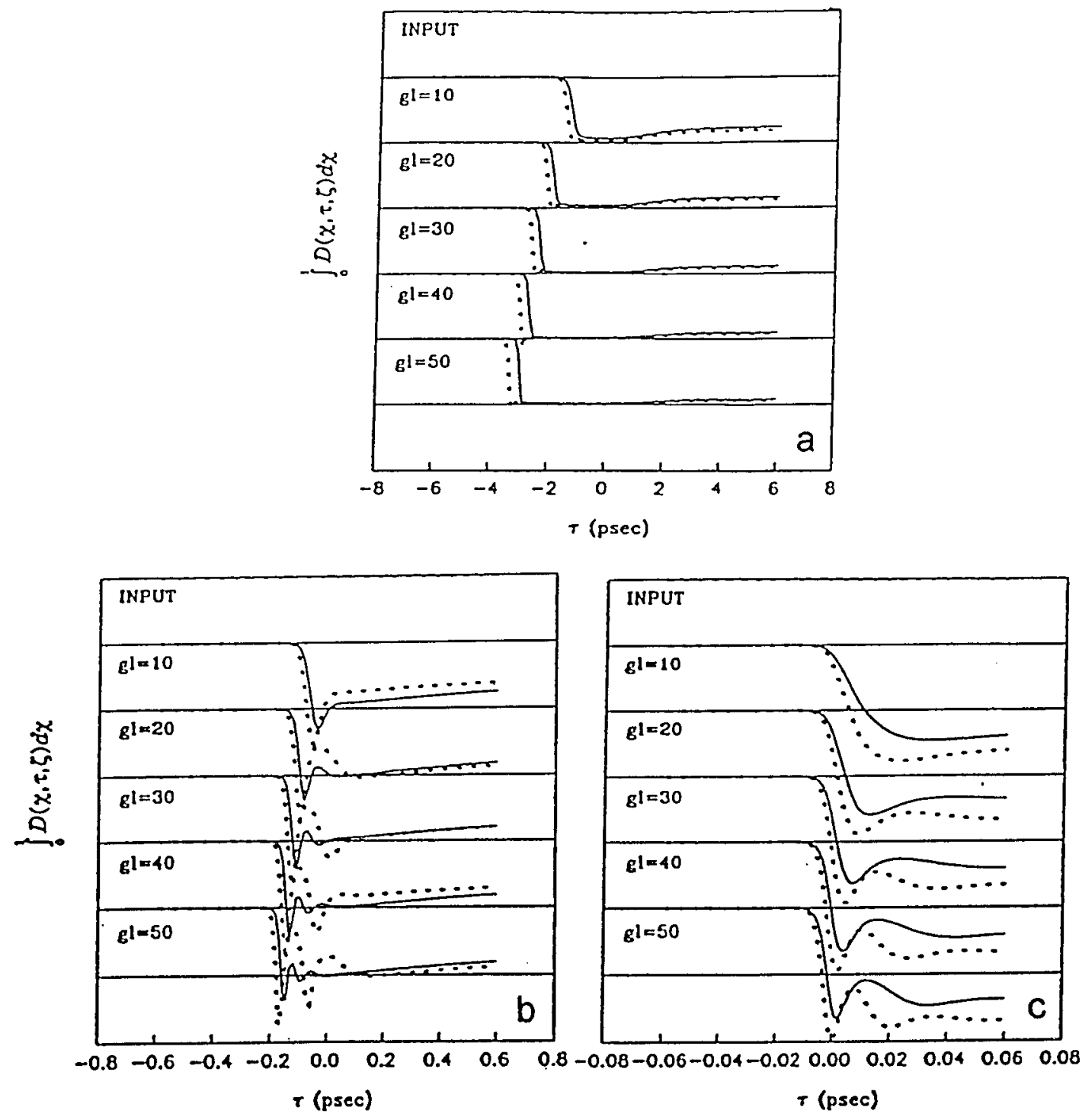

Figure 18. Normalized integrated (over the entire solid angle ) population differences as a function of time. Solid lines are the general semiclassical model (isotropic molecular distribution), and dotted lines are the unidirectional molecular distribution model. The input pulses are (a) $1 \times 10^{-12}$ sec, (b) $1 \times 10^{-13} \mathrm{sec}$, and (c) $1 \times 10^{-14} \mathrm{sec}$. 


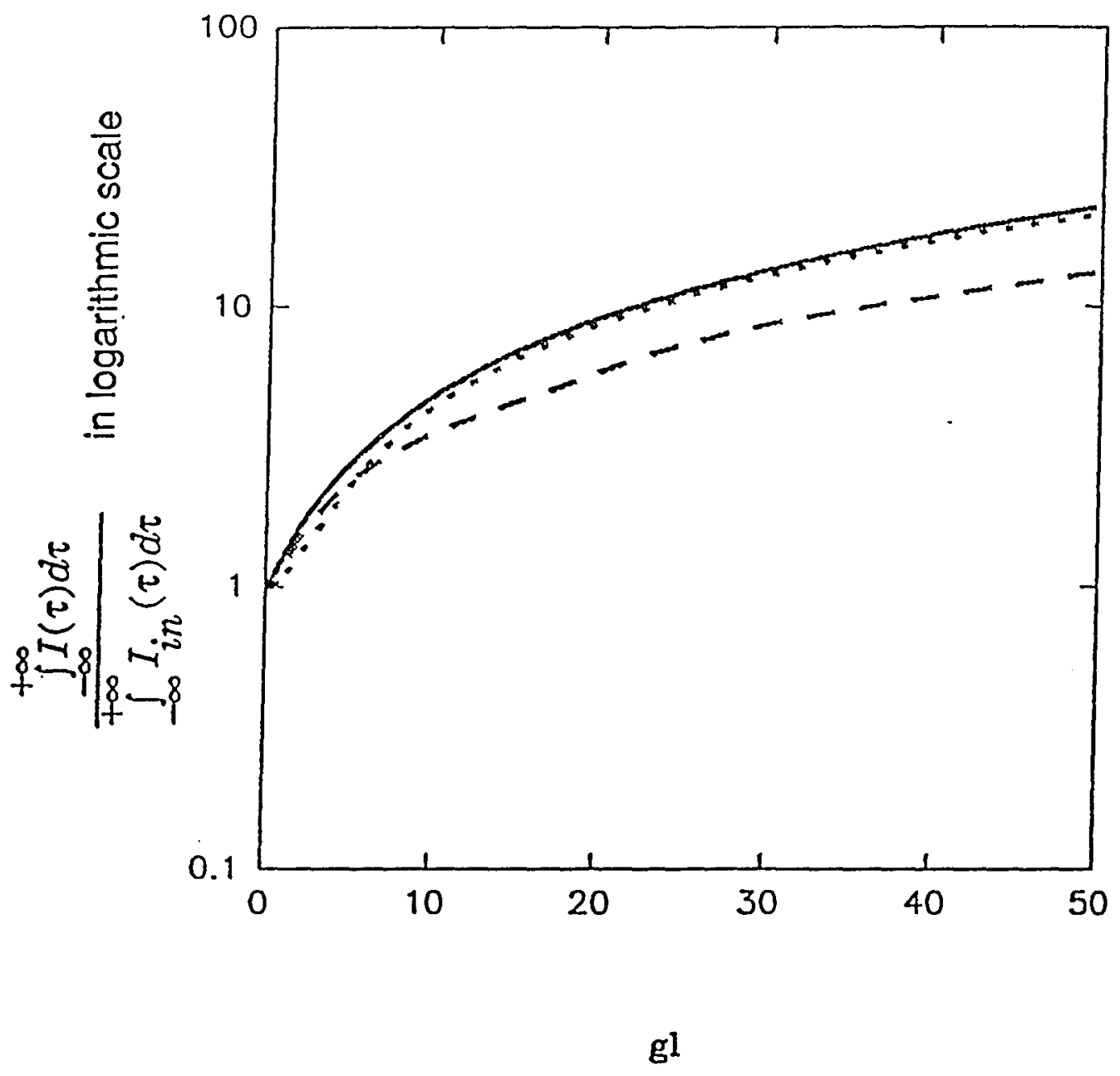

Figure 19. Integrated pulse energy gain based on the unidirectional molecular distribution model of Eqs. (109)-(112) as a function of small signal gain-length product for input pulse widths of $1 \times 10^{-12} \mathrm{sec}$ (solid lines), $1 \times 10^{-13} \mathrm{sec}$ (dashed lines), and $1 \times 10^{-14} \mathrm{sec}$ (dotted lines). Other parameters are the same as in Figure 14. 
studies of the amplifier itself. Such studies are especially needed for subpicosecond and femtosecond pulses where the inaccuracies of the rate equation models are especially conspicuous. The more accurate models will provide the necessary accurate pulse information on parameters such as pulse shape, pulse energy, etc., for subsequent amplifier or absorber stages. Due to the self-compression arising from the coherence time effects in some ranges, the pulse may be directly amplified to high energy without serious pulse broadening and distortion. It should also be noted that models similar to those developed here may be applied to many types of absorbers.

The semiclassical model for the ultrashort dye laser amplifier developed here overcomes the limit of the rate equation approximation of zero coherence time. It is, however, still under the approximation of slowly varying electric field amplitude. Such a treatment is valid as long as the signal transient time is much longer than an optical cycle, which is about $2 \mathrm{fs}$ for a signal at a wavelength of $630 \mathrm{~nm}$. Moreover, due to the nonlinearity of the dye solvents, other nonlinear effects such as self phase modulation and group velocity dispersion should also be considered at very high intensities or in long amplifier cells. 


\section{CHAPTER VI}

\section{CONCLUSIONS}

Generation and amplification of ultrashort optical pulses by dye lasers have gained a lot of interest in science and technology. Shorter and shorter optical pulses with higher and higher energy have been obtained in the past few years, and some quantities related to such optical processes are being pushed to approach the limit of the active dye laser media. Dynamics studies of ultrashort dye lasers require more powerful models to explain the new behaviors of those intense and short optical pulses as well as to exploit the limits of this reliable source of tunable ultrashort optical pulses.

The goal of the dissertation was to extend of the dynamics studies of the ultrashort pulse dye lasers. In particular, we have studied the dynamics of synchronously pumped mode-locked dye lasers, one class of the most reliable sources of ultrashort optical pulses to date, in the range of approaching the limits of active dye media. In addition, amplifications of these ultrashort optical pulses by dye laser amplifiers were modeled with a more comprehensive semiclassical theory. Throughout this work, we have established a self-consistent organization. In Chapter II entitled "Theory of ultrashort pulse dye lasers", general semiclassical models were developed for both dye laser amplifiers and oscillators. These semiclassical models started from Maxwell's wave equation and the density matrix equations, and included a finite coherence time, or phase memory time of the molecular wave function, a finite vibrational relaxation time, and an isotropic molecular orientational distribution. Models derived in this chapter formed the basis for the following studies. 
Chapter IIl entitled "Pump polarization effects in synchronously pumped modelocked dye lasers" showed that the pulsations of a mode-locked dye laser depend strongly on the pump polarization angle. This study took advantage of the semiclassical model which deals with electric field vectors directly, and has investigated the effects on pulse shape, pulse width, pulse intensity etc. of a linearly polarized pump field. This work has been published in the February 15,1991 issue of the Journal of Applied Physics.

In Chapter IV, "Timing and detuning studies of a synchronously pumped modelocked dye laser", used the models developed to investigate the pulse occurrence time as a function of the cavity length mismatch. Such study was found helpful to evaluation of the pulse stability. Experimentally, we have measured the pulse occurrence time relative to the pump pulse. Good agreement was obtained between the theory and experiments. A similar paper based on this chapter may be found in the January 15,1991 issue of Optics Communications.

Chapter V, "Ultrashort pulse propagation in dye laser amplifiers", studied the dynamics associated with ultrashort pulse amplification by dye laser amplifiers. The validity of conventional rate equation models has been examined when applied to ultrashort pulse amplifications. Semiclassical modeling has been found to be important in predicting the pulse amplification, especially the pulse shape. In addition, gain profiles and energy efficiency of dye laser amplifiers were studied. Pulse shortening was predicted by the semiclassical model due to the coherence time effects, which may help the design of multiple stage amplifier chains to obtain desirable pulses. This work may be found in the January 15, 1993 issue of the Journal of Applied Physics.

In general, the semiclassical model developed in this dissertation can apply to dynamics of ultrashort pulses in not only amplifiers but also absorbers. The semiclassical treatment has overcome the limit of the rate equation approximation of zero coherence 
time, and it will be valid as long as the signal transient time is much longer than an optical cycle. Moreover, due to the nonlinearity of the dye solvents, other nonlinear effects such as self phase modulation and group velocity dispersion may play nonnegligible roles especially at very high intensities or in long amplifier cells, and such nonlinear effects may need to be included.

There are several areas which can be pursued further in this work. In the ultrashort pulse amplifier problem, experimental verification was not possible in our research and is still necessary. This will not only test the predictions made by our semiclassical theory but will also help to refine the theoretical models.

Periodic and chaotic pulse train modulation has been found in synchronously pumped mode-locked dye lasers $[66,67]$, but no satisfactory explanation has been given yet. As stable pulses are desired and more useful for practical applications, a better understanding of this phenomenon is important for both the science and technology of these systems. Applying the theoretical models developed in this dissertation and investigating the key parameters responsible for the pulse train instability will be helpful to improving laser performance

Finally, as the semiclassical theory developed in this dissertation is general, one can possibly apply the theory to other ultrashort pulse laser systems as long as the right parameters are used. Such laser systems may include ultrashort pulse solid state lasers [68] and semiconductor lasers [69]. 


\section{REFERENCES}

[1] P. P. Sorokin and J. R. Lankard, "Stimulated emission observed from an organic dye, chloro-aluminum phthalocyanine," IBM Journal of Research and Development, Vol. 10, 162-163 (1966).

[2] L. W. Hillman, "Laser dynamics," in Dye laser principles with applications, edited by F. J. Durate and L. W. Hillman, (New York, 1990), p17.

[3] V. A. Nekhaenko, S. M. Pershin and A. A. Podshivalov, "Synchronously pumped tunable picosecond lasers (review)," Soviet Journal of Quantum Electronics, Vol. $16,299-316(1986)$.

[4] W. H. Glenn, M. J. Brienza and A. J. DeMaria, "Mode locking of an organic dye laser," Applied Physics Letters, Vol. 12, 54-56 (1968).

[5] D. J. Bradley and A. J. F. Durrant, "Generation of ultrashort dye laser pulses by mode locking," Physics Letters A, Vol. 27, 73-74 (1968).

[6] B. H. Soffer and J. W. Lin, "Continuously tunable picosecond pulse organic-dye laser," Joumal of Applied Physics, Vol. 39, 5859-5860 (1968).

[7] C. K. Chan and S. O. Sari, "Tunable dye laser pulse converter for production of picosecond pulses," Electronics Letters, Vol. 25, 403-406 (1974).

[8] J. M. Harris, R. W. Chrisman and F. E. Lytle, "Pulse generation in a cw dye laser by mode-locked synchronous pumping," Applied Physics Letters, Vol. 26, 16-18 (1975).

[9] Z. A. Yasa and O. Teschke, "Picosecond pulse generation in synchronously pumped dye lasers," Optics Communications, Vol. 15, 169-172 (1975).

[10] A. Scavennec and N. S. Nahman, "A simple passively mode-locked CW dye laser," IEEE Journal of Quantum Electronics, Vol. QE-10, 95-96 (1974).

[11] N. J. Frigo, T. Daly and H. Mahr, "A study of a forced mode locked cw dye laser," IEEE Journal of Quantum Electronics, Vol. QE-13, 101-109 (1977). 
[12] C. P. Ausschnitt, R. K. Jain and J. P. Heritage, "Cavity length detuning characteristics of the synchronously mode-locked cw dye laser," IEEE Journal of Quantum Electronics, Vol. QE-15, 915-917 (1979).

[13] J. Hermann and U. Motschmann, "Formation of synchronously mode-locked dye laser pulses," Optics Communications, Vol. 40, 379-384 (1982).

[14] L. M. Davis, J. D. Harvey and J. M. Peart, "Rate equation simulation of a synchronously pumped dye laser," Optics Communications, Vol. 50, 49-55 (1984).

[15] U. Stamm, "Numerical analysis of pulse generation in synchronously mode-locked cw dye lasers," Applied Physics B, Vol. 45, 101-108 (1988).

[16] L. W. Casperson, "Coherence effects in synchronously pumped mode-locked dye lasers," Journal of Applied Physics, Vol. 54, 2198-2208 (1983).

[17] V. Petrov, W. Rudolph and B. Wilhelmi, "Computer simulation of passive modelocking of dye lasers with consideration of coherent light-matter interaction," Optical and Quantum Electronics, Vol. 19, 377-384 (1987).

[18] H. Fu and H. Haken, "Semiclassical theory of dye lasers: the single-frequency and multifrequency steady states of operation," Journal of Optical Society of America $B$, Vol. 5, 899-908 (1988).

[19] D. L. MacFarlane, L. W. Casperson and S. M. Janes, "Coherence effects in hybridly mode-locked dye lasers: theory and experiment," IEEE Journal of Quantum Electronics, Vol. QE-25, 2485-2497 (1989).

[20] B. Zandi, L. W. Casperson and D. L. MacFarlane, "Effects of bandwidth-limiting tuning elements in synchronously pumped mode-locked lasers," Journal of Applied Physics, Vol. 67, 2229-2234 (1990).

[21] F. Kannari, "Multilevel model analysis of energy extraction from a $\mathrm{KrF}$ laser medium by short pulses," Journal of Applied Physics, Vol. 67, 3954-3963 (1990).

[22] F. Kannari and M. Obara, "Multilevel Maxwell-Bloch-equation description of ultrashort laser pulse amplification in inhomogeneously broadened $\mathrm{XeCl}$ media," Journal of Optical Society of America B, Vol. 7, 1493-1506 (1990).

[23] K. E. Hill and G. H. C. New, "Coherent pulse propagation in a $\mathrm{KrF}$ amplifier," Journal of Optical Society of America B, Vol. 8, 839-848 (1991).

[24] P. W. Milonni, R. B. Gibson and A. J. Taylor, "Ultrashort pulse propagation in $\mathrm{KrF}$ laser amplifier," Journal of Optical Society of America B, Vol. 5, 1360-1368 (1988). 
[25] A. M. Johnson and W. M. Simpson, "Tunable femtosecond dye laser synchronously pumped by the compressed second harmonic of Nd:YAG," Journal of Optical Society of America B, Vol. 2, 619-625 (1985).

[26] W. H. Knox, "Femtosecond optical pulse amplification," IEEE Journal of Quantum Electronics, Vol. QE-24, 388-397 (1988).

[27] I. Nagata and T. Nakaya, "Polarization of dye laser light," Journal of Physics D, Vol. 6, 1870-1882 (1973).

[28] S. Jiang and L. W. Casperson, "Pump polarization effects in synchronously pumped mode locked dye lasers," Journal of Applied Physics, Vol. 69, 1866-1871 (1991).

[29] S. Jiang and L. W. Casperson, "Ultrashort-pulse propagation in dye laser amplifiers," Journal of Applied Physics, Vol. 73, (1993).

[30] A. A. Kovalev and V. A. Pilipovich, "Polarization of stimulated emission of organic dyes over the generation spectrum," Bull. Acad. Sci. U.S.S.R., Phys. Ser., Vol. 34, 537-541 (1970).

[31] A. N. Sevchenko, A. A. Kovalev and V. A. Pilipovich, "Dependence of the degree of polarization of induced emission in dyes on the angle between the direction of polarization of the exciting light and the axis of the resonator," Soviet PhysicsDoklady, Vol. 14, 811-813 (1970).

[32] L. G. Pikulik and O. I. Yaroshenko, "Generation kinetics of solutions of complex organic compounds pumped with linearly polarized laser light," Journal of Applied Spectroscopy, Vol. 27, 861-865 (1977).

[33] L. G. Pikulik and O. I. Yaroshenko, "Polarization diagram for dye-based lasers," Journal of Applied Spectroscopy, Vol. 27, 986-990 (1977).

[34] L. I. Burov and I. I. Gancherenok, "Radiation polarization of liquid laser with an isotropic cavity," Optics Spectroscopy (USSR), Vol. 61, 558-560 (1986).

[35] D. W. Phillion, D. Kuizenga and A. E. Siegman, "Rotational diffusion and triplet state processes in dye laser solution," The Journal of Chemical Physics, Vol. 61, 3828-3839 (1974).

[36] K. C. Reyzer and L. W. Casperson, "Polarization characteristics of dye-laser amplifiers I. Unidirectional molecular distribution," Journal of Applied Physics, Vol. $51,6075-6082(1980)$. 
[37] K. C. Reyzer and L. W. Casperson, "Polarization characteristics of dye-laser amplifiers II. Isotropic molecular distribution," Journal of Applied Physics, Vol. 51, 6083-6090 (1980).

[38] A. Dienes, E. P. Ippen and C. V. Shank, "A mode-locked CW dye laser," Applied Physics Letters, Vol. 19, 258-260 (1971).

[39] D. J. Kuizenga, "Mode-locking of the CW dye laser," Applied Physics Letters, Vol. $19,260-263$ (1971).

[40] F. O'Neill, "Picosecond pulses from a passively mode-locked CW dye laser," Optics Communications, Vol. 6, 360-363 (1972).

[41] J. Kuhl, R. Lambrich and D. von der Linde, "Generation of near-infrared picosecond pulses by mode-locked synchronous pumping of a jet-stream dye laser," Applied Physics Letters, Vol. 31, 657-658 (1977).

[42] T. Sizer II, J. D. Kafka, A. Akrisiloff and G. Mourou, "Generation and amplification of sub-picosecond pulse using a frequency-doubled Neodymium YAG Pumping Source," Optics Communications, Vol. 39, 259-262 (1981).

[43] H. A. Haus, "A theory of forced mode locking," IEEE Journal of Quantum Electronics, Vol. QE-11, 323-330 (1975).

[44] D. M. Kim, J. Kuhl, R. Lambrich and D. von der Linde, "Characteristics of picosecond pulses generated from synchronously pumped CW dye laser system," Optics Communications, Vol. 27, 123-126 (1978).

[45] V. A. Nehaenko, "Theory of mode-locked pumping of dye lasers," Soviet Journal of Quantum Electronics, Vol. 11, 446-449 (1981).

[46] A. I. Kovrigin, V. A. Nehaenko, S. M. Pershin and A. A. Podshivalov, "Dynamics of emission from dye lasers pumped synchronously by finite picosecond pulse trains," Soviet Journal of Quantum Electronics, Vol. 14, 1346-1353 (1984).

[47] S. Jiang, D. M. MacFarlane and L. W. Casperson, "Timing and Detuning in Synchronously Pumped Dye Lasers," Optics Communications, Vol. 80, 343-349 (1991).

[48] A. M. Johnson and W. M. Simpson, "Tunable femtosecond dye laser synchronously pumped by the compressed second harmonic of Nd:YAG," Journal of Optical Society of America B, Vol. 2, 619-625 (1985).

[49] T. Urisu and Y. Mizushima, "Cavity length detuning properties in synchronous mode locking," Journal of Applied Physics, Vol. 56, 696-698 (1984). 
[50] D. L. MacFarlane, L. W. Casperson and A. A. Tovar, "Spectral behavior and pulse train instability of a synchronously pumped mode-locked dye laser," Journal of Optical Society of America B, Vol. 5, 1144-1152 (1988).

[51] U. Stamm and F. Weidner, "Pulse formation in synchronously pumped infrared dye lasers," Applied Physics B, Vol. 48, 149-153 (1989).

[52] E. P. Ippen and C. V. Shank, "Subpicosecond spectroscopy," in Picosecond Phenomena, Springer Series in Chemical Physics, Vol. 4, edited by C. V. Shank, E. P. Ippen and S. L. Shapiro (Springer, Berlin, 1978), p103.

[53] R. L. Fork, C. V. Shank and R. Yen, "Amplification of $70 \mathrm{fs}$ optical pulses to gigawatt powers," Applied Physics Letters, Vol. 41, 223-227 (1982).

[54] A. Migus, A. Antonetti, J. Etchepare, D. Hulin and A. Orszag, "Femtosecond spectroscopy with high power tunable optical pulses," Journal of Optical Society of America B, Vol. 2, 584-590 (1985).

[55] C. V. Shank, R. L. Fork, R. Yen, R. H. Stolen and W. J. Tomlinson, "Compression of femtosecond optical pulses," Applied Physics Letters, Vol. 40, 761-762 (1982).

[56] T. L. Gustafson and D. M. Roberts, "A high repetition rate amplifier for the synchronously pumped cavity dumped dye laser," Optics Communications, Vol. 43, 141-144 (1982).

[57] M. C. Downer, R. L. Fork and M. Islam, "3 MHz amplifier for femtosecond optical pulses," in Ultrashort Phenomena IV, edited by D. H. Auston and K. B. Eisenthal (Springer, Berlin, 1984), p27

[58] C. Rolland and P. Corkum, "Amplification of 70 fs pulses in a high repetition rate XeCl pumped dye laser amplifier," Optics Communications, Vol. 59, 64-67 (1986).

[59] D. S. Bethune, "Dye cell design for high power-divergence excimer-pumped dye lasers," Applied Optics, Vol. 20, 1897-1900 (1981).

[60] T. L. Koch, L. C. Chiu and A. Yariv, "Analysis and performance of a picosecond dye laser amplifier chain," Journal of Applied Physics, Vol. 53, 6047-6059 (1982).

[61] A. Migus, J. L. Martin, R. Astier and A. Orszag, "A model of ultra-short pulse amplification," in Fast Phenomena, Vol. IV, p59

[62] A. Migus, C. V. Shank, E. P. Ippen and R. L. Fork, "Amplification of subpicosecond optical pulses," IEEE Journal of Quantum Electronics, Vol. ,QE-18, 101-109 (1982). 
[63] R. T. Leheny and J. Shah, "Amplification and excited state absorption in longitudinally pumped laser dyes," IEEE Journal of Quantum Electronics, Vol. QE11, 70-74 (1975).

[64] U. Ganiel, A. Hatdy, G. Neumann and D. Treves, "Amplified spontaneous emission and signal amplification in dye-laser system," IEEE Journal of Quantum Electronics, Vol. QE-11, 881-892 (1975).

[65] E. O. Schulz-DuBois, "Pulse sharpening and gain saturation in traveling-wave masers," The Bell System Technical Journal, Vol. 43, 625-658 (1964).

[66] D. L. MacFarlane, L. W. Casperson and A. A. Tovar, "Spectral behavior and pulse train instability of a synchronously pumped mode-locked dye laser," Journal of Optical Society of America B, Vol. 5, 1144-1152 (1988).

[67] H. Avramopoulos, P. M. W. French, J. A. R. Williams, G. H. C. New and J. R. Taylor, "Experimental and theoretical studies of complex pulse evolutions in a passively mode-locked ring dye lasers," IEEE Journal of Quantum Electronics, Vol. QE-24, 1884-1892 (1988).

[68] F. Krausz, M. E. Fermann, T. Brabec, P. F. Curley, M. Hofer, M. H. Ober, C. Spielmann, E. Wintner and A. J. Schmidt, "Femtosecond Solid-State Lasers," IEEE Journal of Quantum Electronics, Vol. QE-28, 2097-2121 (1992).

[69] G. P. Agrawal, "Effect of gain dispersion on ultrashort pulse amplification in semiconductor laser amplifiers," IEEE Journal of Quantum Electronics, Vol. 27, 1843-1849 (1991). 